\title{
Determination of Non-Adiabatic Scattering Wave Functions in a Born-Oppenheimer Model
}

\author{
George A. Hagedorn* \\ Department of Mathematics and \\ Center for Statistical Mechanics and Mathematical Physics \\ Virginia Polytechnic Institute and State University \\ Blacksburg, Virginia 24061-0123, U.S.A.
}

\author{
Alain Joye \\ Institut Fourier \\ Unité Mixte de Recherche CNRS-UJF 5582 \\ Université de Grenoble I \\ BP 74 \\ F-38402 Saint Martin d'Hères Cedex, France
}

\begin{abstract}
We study non-adiabatic transitions in scattering theory for the time dependent molecular Schrödinger equation in the Born-Oppenheimer limit. We assume the electron Hamiltonian has finitely many levels and consider the propagation of coherent states with high enough total energy.

When two of the electronic levels are isolated from the rest of the electron Hamiltonian's spectrum and display an avoided crossing, we compute the component of the nuclear wave function associated with the non-adiabatic transition that is generated by propagation through the avoided crossing. This component is shown to be exponentially small in the square of the Born-Oppenheimer parameter, due to the Landau-Zener mechanism. It propagates asymptotically as a free Gaussian in the nuclear variables, and its momentum is shifted. The total transition probability for this transition and the momentum shift are both larger than what one would expect from a naive approximation and energy conservation.
\end{abstract}

\footnotetext{
${ }^{*}$ Partially Supported by National Science Foundation Grants DMS-0071692 and DMS-0303586.
} 


\section{Introduction}

We study scattering theory for the time-dependent molecular Schrödinger equation

$$
i \epsilon^{2} \frac{\partial}{\partial t} \psi(x, t, \epsilon)=\left(-\frac{\epsilon^{4}}{2} \frac{\partial^{2}}{\partial x^{2}}+h(x)\right) \psi(x, t, \epsilon) \quad \text { in } \quad L^{2}\left(\mathbb{R}, \mathbb{C}^{m}\right),
$$

where the electronic hamiltonian $h(x)$ is an $m \times m$ self-adjoint matrix that depends on the nuclear position variable $x \in \mathbb{R}$. The Born-Oppenheimer parameter $\epsilon>0$ denotes the fourth root of the electron mass divided by the mean nuclear mass.

We compute the leading order asymptotics of nuclear wave functions associated with certain non-adiabatic transitions of the electrons. The Landau-Zener mechanism responsible for these makes them exponentially small in $1 / \epsilon^{2}$ as $\epsilon \rightarrow 0$.

Our most general result can be found in Theorem 5.1. Describing the most general situation requires the development of a significant amount of notation and some technical hypotheses. So, in this introduction, we describe two physically interesting special cases that illustrate the main consequences of our analysis in a simple situation. Theorems 6.1 and 6.2 give precise statements of our results for these special cases.

Suppose $h(x)$ is a real $2 \times 2$ self-adjoint matrix that depends analytically on $x$ and has limits $h( \pm \infty)$ as $x \rightarrow \pm \infty$ that are approached sufficiently rapidly. Denote the eigenvalues of $h(x)$ by $e_{j}(x)$, and assume that $e_{2}(x) \geq e_{1}(x)+\delta$ for all $x \in \mathbb{R}$, where $\delta>0$. Near $x=0$, assume $e_{1}$ and $e_{2}$ have an avoided crossing, i.e., $e_{2}(x)-e_{1}(x) \simeq \sqrt{x^{2}+\delta^{2}}$ close to $x=0$, with $\delta$ small but positive. Such an avoided crossing corresponds to complex crossing points $z_{0}$ and $\overline{z_{0}}$, where the analytic continuations of $e_{1}$ and $e_{2}$ satisfy $e_{1}\left(z_{0}\right)=e_{2}\left(z_{0}\right)$, and $z_{0}$ is close to the real axis, with $z_{0}=O(\delta)$.

Let $\phi_{1}(x)$ and $\phi_{2}(x)$ denote normalized, real eigenvectors associated with $e_{1}(x)$ and $e_{2}(x)$.

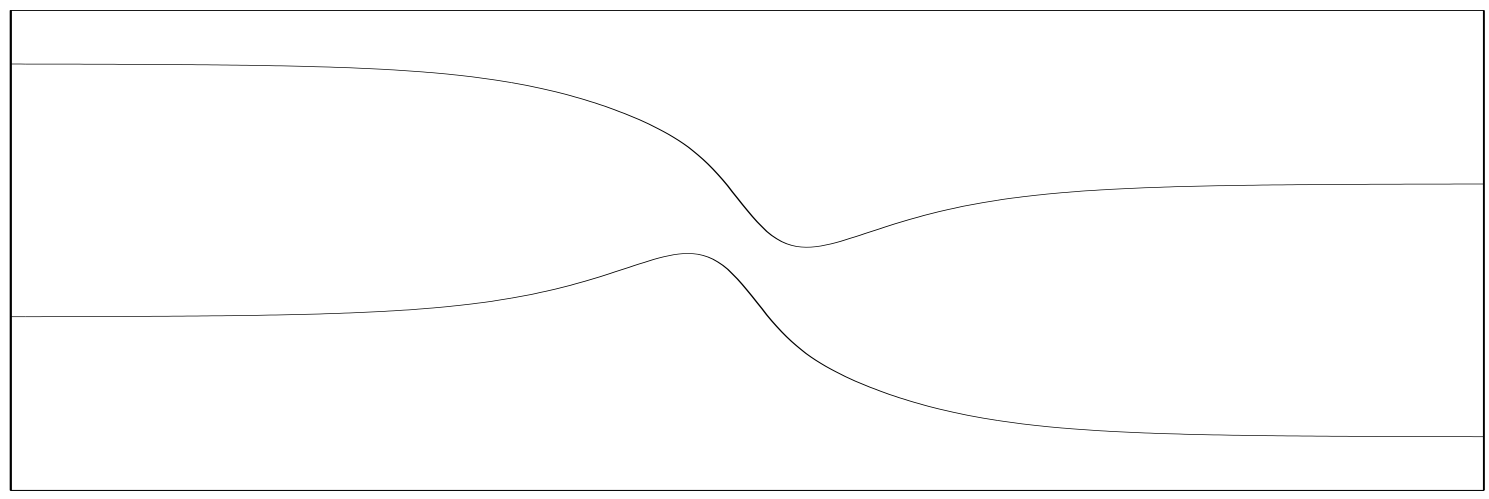

Figure 1. A plot of typical electron energy levels involved in an avoided crossing.

Among the nuclear wave functions we can accommodate are Gaussian coherent states that are defined by

$$
\varphi_{0}\left(A, B, \epsilon^{2}, a, \eta, x\right)=\frac{1}{\pi^{1 / 4} \epsilon^{1 / 2} A^{1 / 2}} \exp \left(-\frac{B(x-a)^{2}}{2 A \epsilon^{2}}+i \frac{\eta(x-a)}{\epsilon^{2}}\right),
$$


where the complex numbers $A$ and $B$ satisfy the normalization condition $\operatorname{Re} \bar{B} A=1$. These states are localized in position near $x=a$, and in momentum near $p=\eta$. Their position uncertainty is $\epsilon|A|$ and their momentum uncertainty is $\epsilon|B|$. For a thorough discussion of these wave packets, see [9].

Choose $E>\sup _{x \in \mathbb{R}} e_{2}(x)$. For a state incoming from the left on the upper electonic level, choose $\eta_{-}>0$. We assume $\eta_{-}$is large enough so that the classical energy $\eta_{-}^{2} / 2+e_{2}(-\infty)>E$. There exists a solution to (1.1) whose large negative $t$ asymptotics are given by

$$
e^{i\left(\eta_{-}^{2} / 2-e_{2}(-\infty)\right) t / \epsilon^{2}} \varphi_{0}\left(A_{-}+i B_{-} t, B_{-}, \epsilon^{2}, a_{-}+\eta_{-} t, \eta_{-}, x\right) \phi_{2}(x),
$$

where the nuclear part is a free Gaussian. Since the electronic levels are isolated from one another, the large positive $t$ asymptotics of this solution are multiples of $\phi_{2}(x)$, up to exponentially small errors in $1 / \epsilon^{2}$. They have the leading behavior determined by the standard time-dependent Born-Oppenheimer approximation as $\epsilon \rightarrow 0$, see [8]:

$$
e^{i \theta_{1}(\epsilon)} e^{i\left(\eta_{1}^{2} / 2-e_{2}(\infty)\right) t / \epsilon^{2}} \varphi_{0}\left(A_{1}+i B_{1} t, B_{1}, \epsilon^{2}, a_{1}+\eta_{1} t, \eta_{1}, x\right) \phi_{2}(x),
$$

where $e^{i \theta_{1}(\epsilon)}$ is some explicit phase, and the parameters $A_{1}, B_{1}, a_{1}, \eta_{1}$ are determined by the scattering properties of the classical Hamiltonian $p^{2} / 2+e_{2}(x)$.

Our interest lies with the leading order asymptotics of the non-adiabatic component of the wave function for large positive $t$ and $\epsilon \rightarrow 0$. We prove in Theorem 6.1 that these have the form

$$
c_{0} e^{-\alpha^{*} / \epsilon^{2}} e^{i \theta_{+}(\epsilon)} e^{i\left(\eta_{+}^{2} / 2-e_{1}(\infty)\right) t / \epsilon^{2}} \varphi_{0}\left(A_{+}+i B_{+} t, B_{+}, \epsilon^{2}, a_{+}+\eta_{+} t, \eta_{+}, x\right) \phi_{1}(x),
$$

and we specify how the phase $\theta_{+}(\epsilon)$, the $\epsilon$-independent amplitude $c_{0}>0$, the exponential decay rate $\alpha^{*}>0$, and the parameters of the free Gaussian part $A_{+}, B_{+}, a_{+}$, and $\eta_{+}>0$ are determined. As a corollary, the leading term of the transition amplitude $\mathcal{A}(\epsilon)$ (whose absolute square is the transition probability) is given by the quantity

$$
\mathcal{A}(\epsilon)=c_{0} e^{i \theta_{+}(\epsilon)} e^{-\alpha^{*} / \epsilon^{2}}, \quad \text { as } \quad \epsilon \rightarrow 0 .
$$

Let us describe the main features of this exponentially small transmitted part of the wave function. One may naively expect $\eta_{+}$to be determined by the energy conservation condition

$$
\frac{\eta_{-}^{2}}{2}+e_{2}(-\infty)=\frac{\eta_{+}^{2}}{2}+e_{1}(\infty),
$$

but this yields the wrong value. The correct value is larger. Intuitively, this is due to the faster parts of the wave function behaving less adiabatically than the slower parts. Because this dependence on the speed appears in an exponent, it leads to an $O(1)$ change in the final momentum $\eta_{+}$. In other words, the higher momentum components of the incoming state are much more likely to make a transition than the lower momentum components. Hence, after the transition, there are more fast pieces of the wave function, and the final average mometum is greater than one would naively expect from an energy conservation calulation based solely on the average incoming momentum.

This also affects the transition amplitude which is larger than what is naively expected. It is asymptotically composed of an $\epsilon$-independent prefactor $c_{0}$ times an exponentially small 
quantity $e^{-\alpha^{*} / \epsilon^{2}}$, whose decay rate $\alpha^{*}$ is related to that of the Landau-Zener decay rate for purely adiabatic problems. Actually, $\alpha^{*}$ consists of the sum of the imaginary part of some action integral around the complex electronic eigenvalue crossing point $z_{0}$ and a contribution that depends explicitly on the nuclear part of initial incoming state (1.2). The action

integral depends only on the electronic levels and reads $\int_{\zeta} \sqrt{2\left(E-e_{2}(z)\right.} d z$ where $\zeta$ is a loop in the complex plane based at the origin encircling $z_{0}$. The contribution from the nuclear part of the wave packet depends on the shape of its momentum/energy density. It is that last contribution that makes the obvious candidate given by the imaginary part of the action integral taken at the classical energy $E$, miss the actual value of the decay rate $\alpha^{*}$. In that sense, (1.3), which we could call a molecular Landau-Zener formula, cannot be determined from the usual adiabatic Landau-Zener formula with just the knowledge of the electronic levels and the classical nuclear momentum close to the avoided crossing. Indeed, our analysis shows that we also need to take into account the details of the incoming wave packet to determine (1.3). This is why we resort to coherent states to get such accurate asymptotics.

The way we obtain all our results is by employing a time-independent scattering theory approach that uses generalized eigenfunctions of the full Hamiltonian. We expand the wave function in terms of the generalized eigenfunctions and calculate the large $|t|$ asymptotics. For every incoming momentum $k$ there is classical energy conservation, but a different probability of making the non-adiabatic transition. We obtain the correct $\alpha^{*}$ and $\eta_{+}$by computing the averages over $k$ rather than by doing one calculation based on the average incoming momentum $\eta_{-}$.

\section{Remarks}

1. We obtain the analogous results when the incoming state is associated with the lower electronic level $e_{1}$, provided that we keep the average total energy above both the levels.

2. There are other components of the scattered wave function. For example, one should expect a reflected wave on the $e_{2}$ electronic level and also a reflected wave on the $e_{1}$ level. We prove that if the avoided crossing has a sufficiently small gap, then the other components are exponentially even smaller in $1 / \epsilon^{2}$ than the transmitted non-adiabatic term we compute.

The second situation we describe in this introduction involves the same set-up as above, but with the Gaussian incoming states replaced by more general incoming coherent states. This example illustrates the second key feature that our analysis demonstrates: even if the incoming state is not Gaussian, the outgoing non-adiabatic transition state, generically, is Gaussian to leading order in $\epsilon$.

For $m=1,2, \ldots$, we define

$$
\begin{aligned}
\varphi_{m}\left(A, B, \epsilon^{2}, a, \eta, x\right) & = \\
& 2^{-m / 2}(m !)^{-1 / 2} A^{-m / 2}(\bar{A})^{m / 2} H_{m}\left(\frac{x-a}{\epsilon|A|}\right) \phi_{0}\left(A, B, \epsilon^{2}, a, \eta, x\right),
\end{aligned}
$$

where $H_{m}$ is the $m^{\text {th }}$ order Hermite polynomial.

We now replace (1.2) by

$$
e^{i\left(\eta_{-}^{2} / 2-e_{2}(-\infty)\right) t / \epsilon^{2}} \varphi_{m}\left(A_{-}+i B_{-} t, B_{-}, \epsilon^{2}, a_{-}+\eta_{-} t, \eta_{-}, x\right) \phi_{2}(x) .
$$


Again, up to exponentially small errors, the large positive $t$ asymptotics of the solution are multiples of $\phi_{2}(x)$. Their leading behavior is determined by the standard time-dependent Born-Oppenheimer approximation,

$$
e^{i \theta_{1}(\epsilon)} e^{i\left(\eta_{1}^{2} / 2-e_{2}(\infty)\right) t / \epsilon^{2}} \varphi_{m}\left(A_{1}+i B_{1} t, B_{1}, \epsilon^{2}, a_{1}+\eta_{1} t, \eta_{1}, x\right) \phi_{2}(x),
$$

where $A_{1}, B_{1}, a_{1}, \eta_{1}$, and $\theta_{1}(\epsilon)$ are the same as in our first example. However, our Theorem 6.2 shows that the leading order asymptotics of the non-adiabatic component of the wave function for large positive $t$ again have the form of a freely propagating Gaussian

$$
c_{m} \epsilon^{-m} e^{-\alpha^{*} / \epsilon^{2}} e^{i \theta_{+}(\epsilon)} e^{i\left(\eta_{+}^{2} / 2-e_{1}(\infty)\right) t / \epsilon^{2}} \varphi_{0}\left(A_{+}+i B_{+} t, B_{+}, \epsilon^{2}, a_{+}+\eta_{+} t, \eta_{+}, x\right) \phi_{1}(x),
$$

and display a pre-exponential factor of order $\epsilon^{-m}$. The values of $\alpha^{*}, A_{+}, B_{+}, a_{+}$, and $\eta_{+}$are the same as in our first example, and we determine the prefactor $c_{m}$. The numerics presented below clearly illustrate these features.

Our most general result, Theorem [5.1, extends these results in several ways. First, we can handle electron Hamiltonians $h(x)$ that are $m \times m$ complex hermitian matrices which have two levels of interest that have an avoided crossing. These levels must stay well separated from the rest of the spectrum of $h(x)$. Second, we can handle situations in which several levels display certain patterns of avoided crossings. For example, when two levels have an avoided crossing for one value of $x$, and one of those levels has another avoided crossing with a third level for some other value of $x$. However, in such cases, we can only study the non-adiabatic components for certain levels. The ones we can handle depend on the order in which the levels have the avoided crossings. Third, we can consider more general incoming states that do not have the form of the $\varphi_{j}$ 's considered above. They are characterized by an energy (or momentum) distribution which is sharply peaked around some fixed energy, so that a semiclassical analysis can be performed. In such general cases also, the nuclear part of the non-adiabatic wave function is Gaussian and exponentially small, with a decay rate sharing the properties described above.

The paper is organized as follows: In the rest of the Introduction, we review the relevant literature and present numerical results for the above examples. They show excellent agreement with our analysis. In Section 2 , we set up the general problem we study. We state most of our hypotheses here and make precise the notion of avoided crossing. In Section 3, we study generalized eigenvectors of the full Hamiltonian. In particular, their WKB-type analysis in the complex plane is performed here. We superimpose the generalized eigenvectors to generate solutions to the time-dependent Schrödinger equation and construct asymptotic scattering states in Section 4. Non-adiabatic transition asymptotics are studied in Section 5, where our most general result is stated as Theorem 5.1. Further properties and estimates on the energy and momentum shifts are provided in Section 5. Section 6 is devoted to the special case of interest where the nuclear part of the incoming state is a Gaussian or a Gaussian times a Hermite polynomial as in (1.5). Finally, Section 7 contains the proofs of several technical results that are stated in the earlier sections.

From this outline, one can see that our results depend crucially on the properties of generalized eigenvectors of the full Hamiltonian. We prove these properties by applying the 
ideas and results of Joye [14, [15] that provide exponentially accurate WKB-type results in a generic avoided-crossing regime, generalizing earlier two-level adiabatic techniques from [17, [18, [19]. See also [21], 25] for stationary results of the same kind. That a complex WKB-type analysis plays an important role here should be no surprize. Indeed, in the ODE context of adiabatic-like problems dealt with in the references above, the complex WKB approach proved to be the most efficient method providing a quantitative analysis of the exponentially small leading order term of the Landau-Zener mechanism. See, however, [13] and 2] for a different successful approach of such problems, based on optimal truncation techniques.

There are mathematical results on the exponentially small size of non-adiabatic transitions in the Born-Oppenheimer approximation, and for related problems. See, e.g., [12], [23], 1], 24], 22. However, to the best of our knowledge, there are no rigorous results on this topic in the literature that actually compute the leading asymptotics of non-adiabatic transitions in our time-dependent PDE setting. We have recently learned that Betz and Teufel, 3], are adapting techniques from [2] to the Born-Oppenheimer setup. They have formal and numerical results for specific electronic hamiltonians in agreement with ours. Also, rigorous results on the propagation of wave packets through avoided crossings, representing first attempts to unravel the molecular Landau-Zener mechanism, are obtained in [10], [11]. (See also [26.) In those papers, the gap $\delta$ shrinks to zero with $\epsilon$ in such a way that the transitions are of order one, so that they can be computed by perturbation theory. This is in contrast to the present situation, in which $\delta$ is small but fixed as $\epsilon \rightarrow 0$, and the transitions are exponentially small.

Because of the importance of the Landau-Zener mechanism to molecular physics, there are relevant papers in the physics and chemistry literature. See, e.g., [4, [27], 28].

\subsection{Numerical Simulations for a Gaussian Initial State}

We now present graphical results of a numerical simulation in which the initial state is a Gaussian function associated with the upper energy level for a two level system. These plots are in very good agreement with the results of our analysis.

We have numerically integrated equation (1.1) with $\epsilon=0.2$ for the Hamiltonian function

$$
h(x)=\frac{1}{2}\left(\begin{array}{cc}
1 & \tanh (x) \\
\tanh (x) & -1
\end{array}\right) .
$$

The energy levels are $\pm \frac{1}{2} \sqrt{1+\tanh (x)^{2}}$, and there is an avoided crossing at $x=0$ with a minimum gap of 1 . The initial state is the eigenvector associated with the upper energy level times the Gaussian $\phi_{0}\left(A_{0}+i t B_{0}, B_{0}, \epsilon^{2}, \eta t, \eta, x\right)$, where $A_{0}=B_{0}=\eta=1$, with the initial time $t=-10$. The following two figures show the initial position and momentum probability densities, respectively. In both plots, the probablity of being on the lower energy level is zero. 


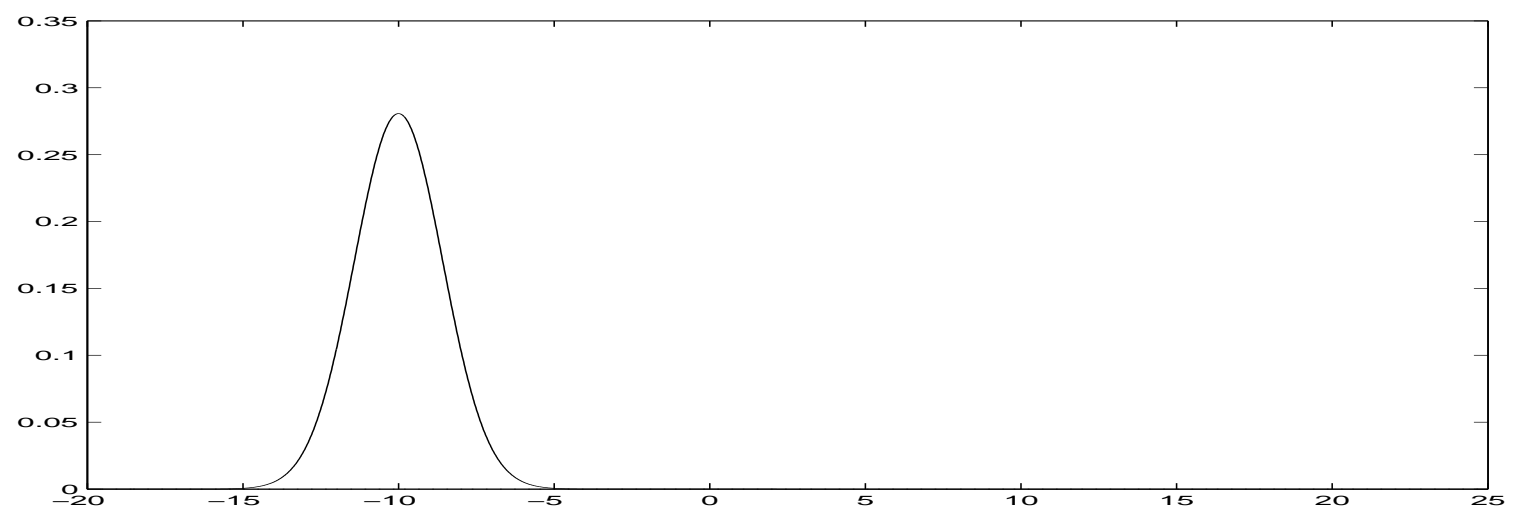

Figure 2. Position space plot at time $t=-10$ of the probability density for being on the upper energy level (solid line), and $3 \times 10^{8}$ times the probability density for being on the lower energy level (dotted line).

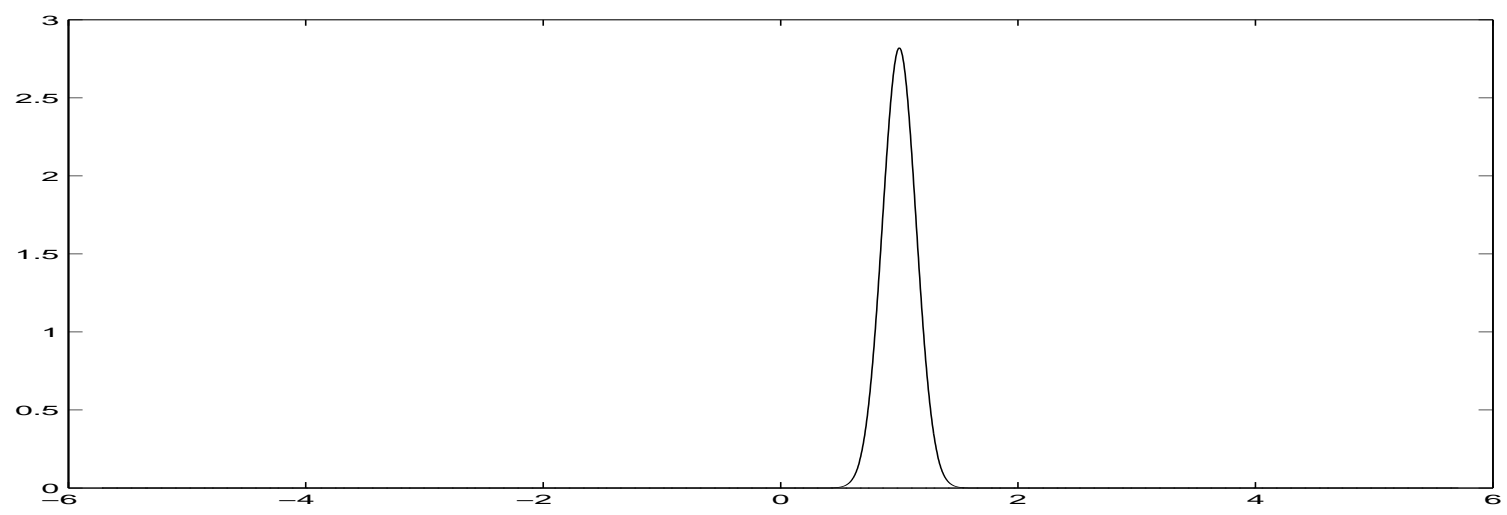

Figure 3. Momentum space plot at time $t=-10$ of the probability density for being on the upper energy level (solid line), and $3 \times 10^{8}$ times the probabilty density for being on the lower energy level (dotted line).

The following two plots show the position and momentum probability densities at $t=9$ after the wave function has interacted with the avoided crossing. The component associated with the lower energy level has mean momentum 2.05. It is evident from the plot that it is greater than 2 .

The naive energy conservation calculation predicts the following: The total energy is $E=\eta^{2} / 2+1 / 2 \sqrt{1+\tanh (-10)^{2}}=1.2071$. After the transition to the lower surface, the kinetic energy should be this value plus $\sqrt{2} / 2$, so $\eta_{1}^{2} / 2=1.9142$. This predicts a final momentum after the transition of $\eta_{1}=1.9566$. 


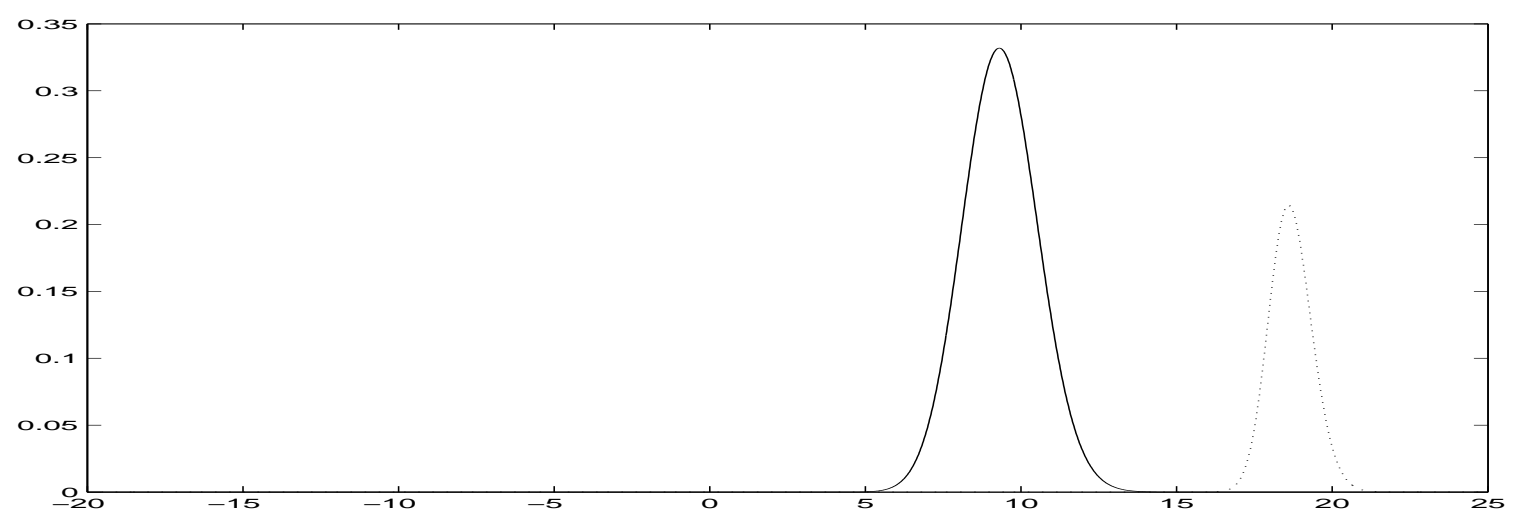

Figure 4. Position space plot at time $t=9$ of the probability density for being on the upper energy level (solid line), and $3 \times 10^{8}$ times the probabilty density for being on the lower energy level (dotted line).

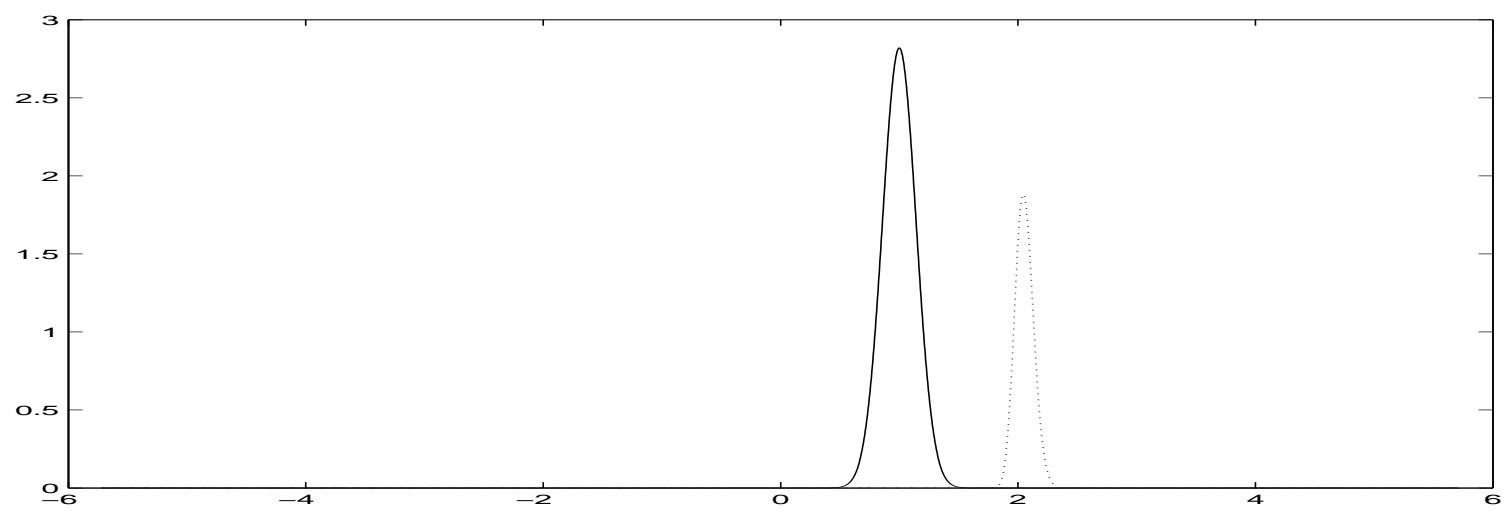

Figure 5. Momentum space plot at time $t=9$ of the probability density for being on the upper energy level (solid line), and $3 \times 10^{8}$ times the probabilty density for being on the lower energy level (dotted line).

\subsection{Numerical Simulations for More General Initial States}

We next present the results for the same system as above, but where the initial Gaussian $\phi_{0}$ has been replaced by $\phi_{3}$. See (1.4). Note that the transition amplitude is significantly larger than in the example above, and that the component of the wave function that makes the transition to the lower level is approximately a Gaussian. The value of epsilon $\epsilon=0.2$ is not particularly small, so the component of the final state that does not make a transition is only approximately a $\phi_{3}$ wave packet. We have chosen this relatively large value of epsilon to avoid numerical difficulties in integrating equation (1.1).

We should also note that the naive energy conservation calculation again predicts that the component of the wave function on the lower level should have mean momentum 1.9566. Since initial wave function has a greater momentum uncertainty than in the Gaussian example above, we see an even greater discrepancy between this prediction and the correct value. Our simulation yields a value of roughly 2.25 . 


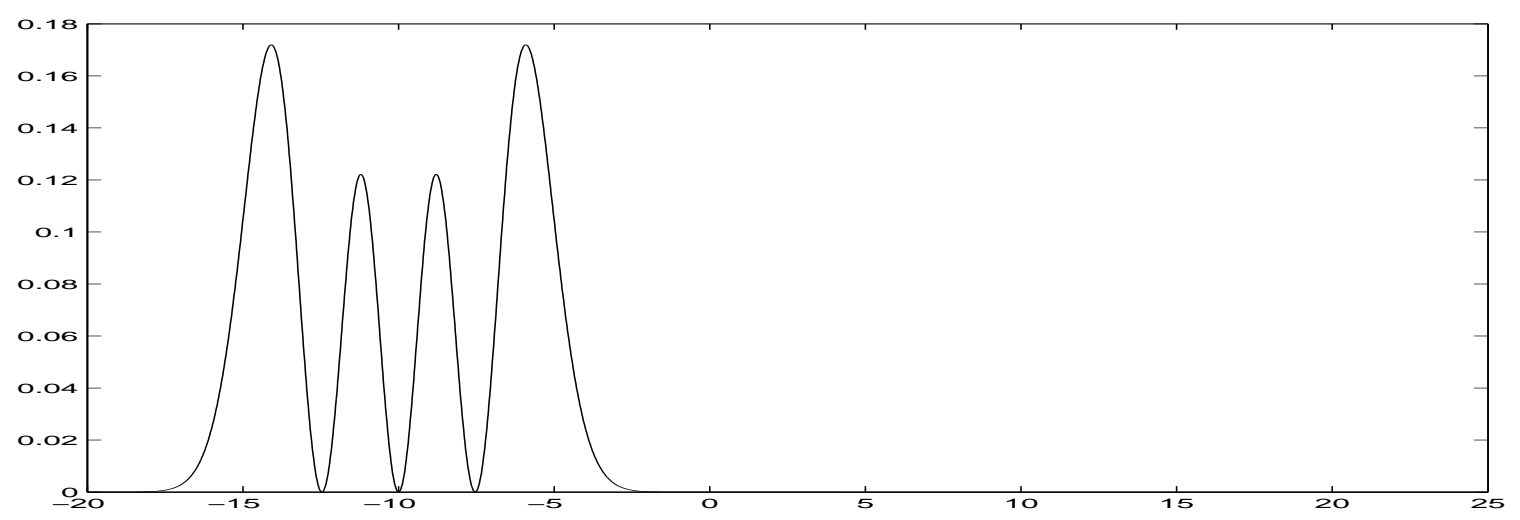

Figure 6. Position space plot at time $t=-10$ of the probability density for being on the upper energy level (solid line), and $10^{7}$ times the probabilty density for being on the lower energy level (dotted line).

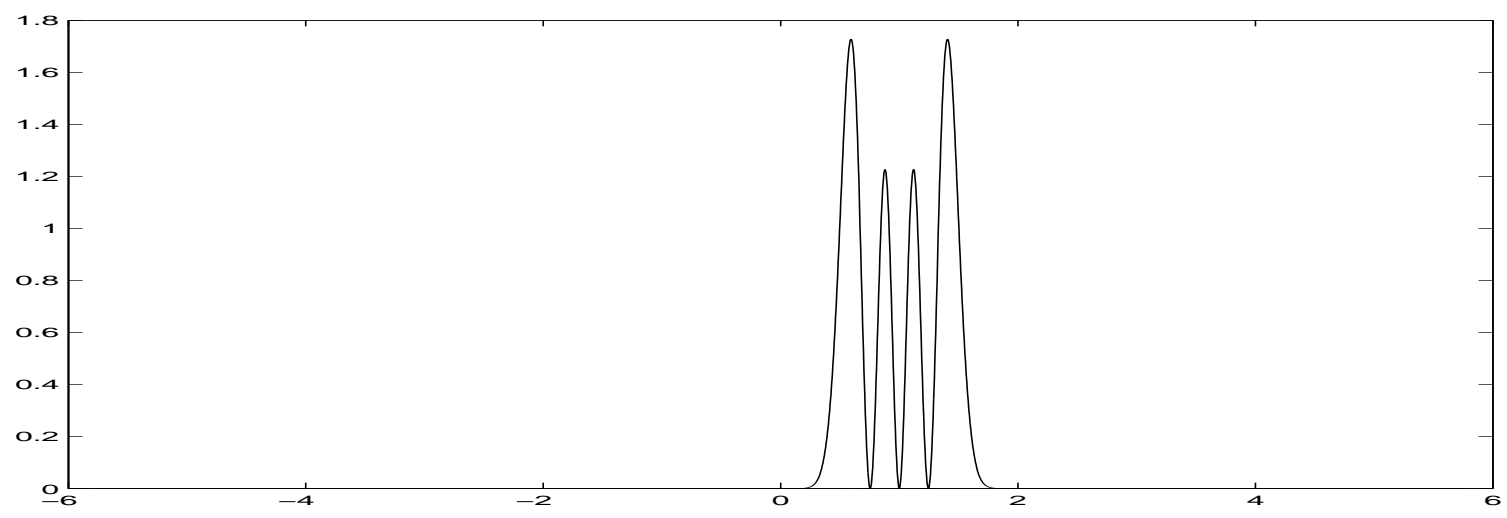

Figure 7. Momentum space plot at time $t=-10$ of the probability densityfor being on the upper energy level (solid line), and $10^{7}$ times the probabilty density for being on the lower energy level (dotted line).

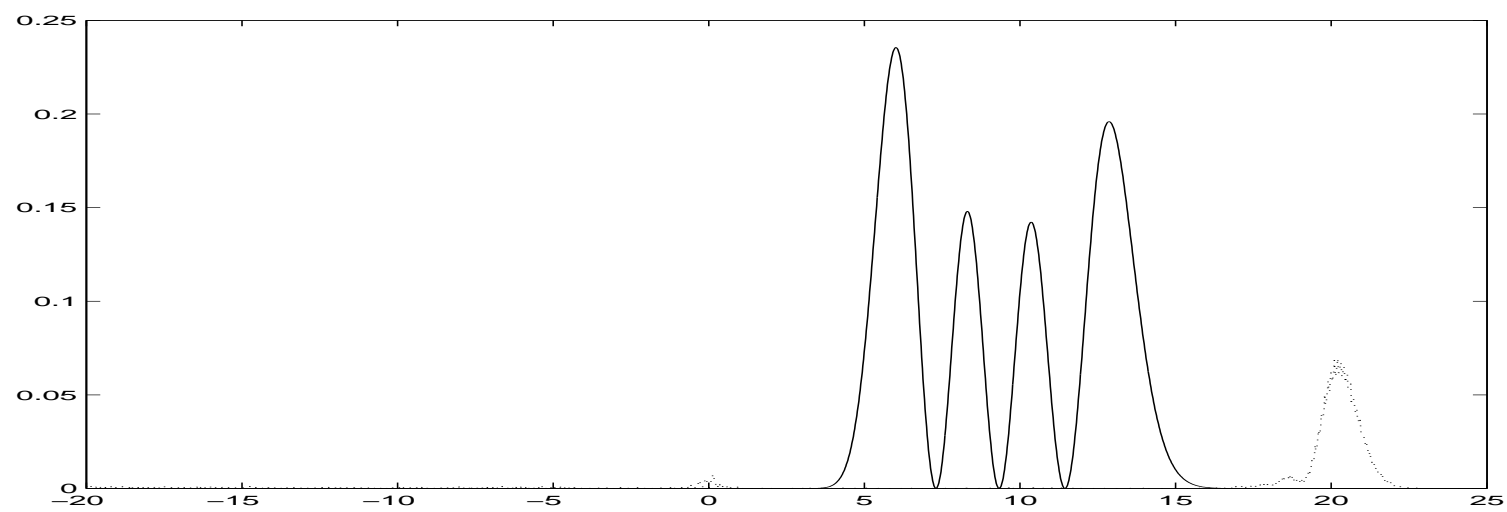

Figure 8. Position space plot at time $t=9$ of the probability density for being on the upper energy level (solid line), and $10^{7}$ times the probabilty density for being on the lower energy level (dotted line). 


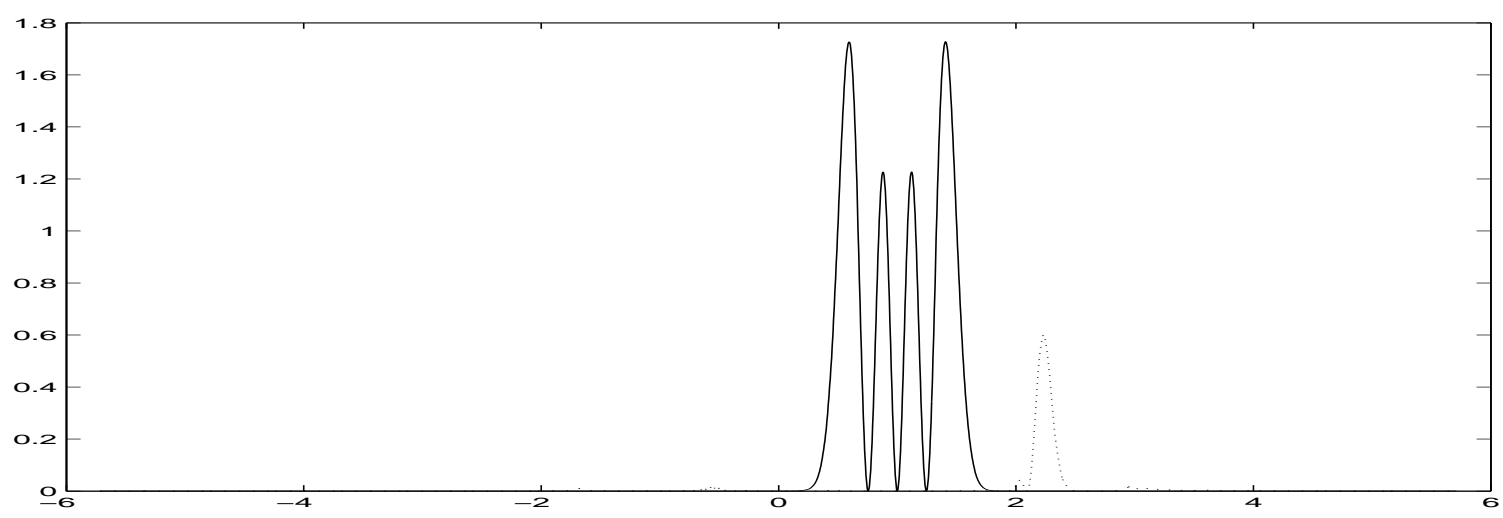

Figure 9. Momentum space plot at time $t=9$ of the probability density for being on the upper energy level (solid line), and $10^{7}$ times the probabilty density for being on the lower energy level (dotted line).

Acknowledgements George Hagedorn wishes to thank the Institut Fourier and the City of Grenoble for their kind hospitality and support during 2003 and 2004 when this research was conducted.

\section{Hypotheses for the Electron Hamiltonian}

We begin with three general assumptions about the electron Hamiltonian $h$. We then impose two more assumptions that make precise the avoided crossing situations we can handle.

H1: We assume $z \mapsto h(z)$ is a $m \times m$ matrix-valued analytic function that is analytic in $z \in \rho_{\alpha}=\{z=x+i y:|y| \leq \alpha\}$, where $\alpha>0$. We assume $h(z)$ is self-adjoint for $z \in \mathbb{R}$.

Since we work in a scattering framework, we further assume:

H2: There exist $\nu>1 / 2, c$, and two matrices $h( \pm \infty)$, such that for all $x \in \mathbb{R}$,

$$
\sup _{|y| \leq \alpha}\|h(x+i y)-h( \pm \infty)\| \leq \frac{c}{<x>^{2+\nu}}
$$

where $\left\langle x>\right.$ denotes $\left(1+x^{2}\right)^{1 / 2}$.

The rate of convergence in this assumption can certainly be weakened. However, general scattering theory is not the main point of the present study.

H3: We assume the spectrum $\sigma(h(x))$ of $h(x)$ consists of $m$ non-degenerate eigenvalues

$$
\sigma(h(x))=\left\{e_{j}(x)\right\}_{j=1, \cdots, m},
$$

for any $x \in \mathbb{R} \cup\{ \pm \infty\}$.

We let $\phi_{j}(x), j=1, \cdots, m$, denote the corresponding eigenvectors, characterized up to constant phases by the following conditions

$$
\left\|\phi_{j}(x)\right\| \equiv 1, \quad \text { and } \quad\left\langle\phi_{j}(x), \phi_{j}^{\prime}(x)\right\rangle \equiv 0, \quad \forall j=1, \cdots, m,
$$


where the the prime denotes the derivative with respect to $x$. The eigenvectors are analytic in some narrow open strip containing the real axis [20].

By using the Cauchy formula, it is easy to check that our hypotheses imply

$$
\frac{d^{n}}{d x^{n}}\left(e_{j}(x)-e_{j}( \pm \infty)\right)=O\left(<x>^{-(2+\nu)}\right)
$$

and

$$
\frac{d^{n}}{d x^{n}}\left(\phi_{j}(x)-\phi_{j}( \pm \infty)\right)=O\left(<x>^{-(2+\nu)}\right)
$$

for any $n \in \mathbf{N}$.

We now make specific assumptions concerning avoided crossings for $h$.

The idea is to assume $h(x)$ belongs to a smooth family of electron Hamiltonians $h(x, \delta)$. When $\delta=0$, we assume there are actual crossings. When $\delta \neq 0$, we assume there are no crossings for real values of $x$. The electron Hamiltonians we actually use have the form $h(x, \delta)$ for some small, but fixed value of $\delta$.

Our precise assumption is the following:

H4: For each fixed $\delta \in[0, d]$, the matrix $h(x, \delta)$ satisfies $\mathbf{H 1}$ in a strip $\rho_{\alpha}$ independent of $\delta$, and $h(z, \delta)$ is $C^{2}$ as a function of the two variables $(z, \delta) \in \rho_{\alpha} \times[0, d]$. Moreover, $h(\cdot)$ satisfies $\mathbf{H} 2$ uniformly for $\delta \in[0, d]$, with limiting values $h( \pm \infty, \delta)$ that are $C^{2}$ functions of $\delta \in[0, d]$.

Again, some of our results hold under weaker smoothness assumptions.

We can deal with multiple avoided crossings, but cannot deal with all possible patterns of avoided crossings. The following assumption describes the ones we allow:

H5: For each $x \in \mathbb{R}$ and each $\delta \in[0, d], \sigma(h(x, \delta))$ consists of $m$ real eigenvalues

$$
\sigma(h(x, \delta))=\left\{e_{1}(x, \delta), e_{2}(x, \delta), \cdots, e_{m}(x, \delta)\right\} \subset \mathbb{R} .
$$

When $\delta>0$ we assume these are distinct for $x \in[-\infty,+\infty]$ and are labeled by

$$
e_{1}(x, \delta)<e_{2}(x, \delta)<\cdots<e_{m}(x, \delta) .
$$

When $\delta=0$, the eigenvalues are $m$ analytic functions that have finitely many real crossings at $x_{1} \leq x_{2} \leq \cdots \leq x_{p}$, with $p \geq 1$. We assume the eigenvalues have $m$ distinct limits as $x \rightarrow-\infty$ and as $x \rightarrow \infty$. We label these eigenvalues $e_{j}(x, 0)$ in a way that is discontinuous in $\delta$ near $\delta=0$. This labeling is determined by the following conditions:

i) For all $x<x_{1}$,

$$
e_{1}(x, 0)<e_{2}(x, 0)<\cdots<e_{m}(x, 0) .
$$

ii) For all $j<l \in\{1,2, \cdots, n\}$, there exists at most one $x_{r}$ with

$$
e_{j}\left(x_{r}, 0\right)-e_{l}\left(x_{r}, 0\right)=0,
$$

and if such an $x_{r}$ exists, we have

$$
\frac{\partial}{\partial x}\left(e_{j}\left(x_{r}, 0\right)-e_{l}\left(x_{r}, 0\right)\right)>0 .
$$


iii) For all $j \in\{1,2, \cdots, n\}$, the eigenvalue $e_{j}(x, 0)$ crosses eigenvalues whose indices are all superior to $j$ or all inferior to $j$.

\section{Remarks:}

i) The parameter $\delta$ can be understood as a coupling constant that controls the strength of the perturbation that lifts the degeneracies of $h(x, 0)$ on the real axis.

ii) Analyticity of the eigenvalues $e_{j}(x, 0)$ on the real axis follows from the self-adjointness of $h(x, 0)$.

iii) The crossings are assumed to be generic in the sense that the derivatives of $e_{j}-e_{k}$ are non-zero at the crossing $x_{r}$. This ensures that when $\delta>0$ is small, the generic behavior (3.19) holds at the corresponding complex crossing points.

iv) When $m=2, \mathbf{H} 5$ requires that the two eigenvalues have exactly one generic crossings when $\delta=0$.

v) The crossing points $\left\{x_{1}, x_{2}, \cdots, x_{p}\right\}$ need not be distinct, which is important when the Hamiltonian possesses symmetries. However, for each $j=1, \cdots, n$, the eigenvalue $e_{j}(x, \delta)$ experiences avoided crossings with $e_{j+1}(x, \delta)$ and/or $e_{j-1}(x, \delta)$ at a subset of distinct points $\left\{x_{r_{1}}, \cdots, x_{r_{j}}\right\} \subseteq\left\{x_{1}, x_{2}, \cdots, x_{p}\right\}$.

For certain results, we also impose the condition that these avoided crossings be generic in the sense of [7] and [14. This condition essentially says that the low order Taylor series coefficients of certain quantities do not vanish at the crossing when $\delta=0$.

H6: Near an avoided crossing of $e_{j}(x, \delta)$ and $e_{n}(x, \delta)$, there exist $a>0, b>0$, and $c \in \mathbb{R}$, such that

$$
e_{n}(x, \delta)-e_{j}(x, \delta)= \pm \sqrt{a x^{2}+2 c x \delta+b^{2} \delta^{2}+R_{3}(x, \delta)},
$$

where $c^{2}<a^{2} b^{2}$ and $R_{3}(x, \delta)$ is a remainder of order 3 in $(x, \delta)$ close to $(0,0)$.

Our final hypothesis involves both the electron Hamiltonian and an interval of energies, $\Delta$. We ultimately consider states of the full Hamiltonian whose energy is concentrated in $\Delta$, with $\Delta$ high enough that scattering onto all the electron energy levels is possible. An energy range that satisfies this condition can always be chosen for some strip $\rho_{\alpha}$, provided the minimum value in $\Delta$ is large enough.

H7: The interval $\Delta \in \mathbb{R}$ is compact and has non-empty interior. Furthermore, it is chosen so that

$$
\inf _{\substack{E \in \Delta \\ z \in \rho_{\alpha} \\ \delta \in[0, \delta]}}\left|E-e_{j}(z, \delta)\right|>0
$$

\section{$3 \quad$ Generalized Eigenvectors}

For energies $E \in \Delta$, we construct generalized eigenvectors for the full Hamiltonian. For the time being, the parameter $\delta>0$ is fixed and we drop it in the notation. The generalized eigenvectors are solutions $\Psi(x, E, \epsilon) \in \mathbb{C}^{m}$ to the time-independent Schrödinger equation

$$
\left(-\frac{\epsilon^{4}}{2} \frac{\partial^{2}}{\partial x^{2}}+h(x)\right) \Psi(x, E, \epsilon)=E \Psi(x, E, \epsilon) .
$$


For each $E \in \Delta$, the set of such solutions is $2 m$-dimensional, and individual solutions can be characterized by their asymptotics at $x=-\infty$ (or at $x=\infty)$.

Let

$$
\Phi(x, E, \epsilon)=\left(\begin{array}{c}
\Psi(x, E, \epsilon) \\
i \epsilon^{2} \frac{\partial}{\partial x} \Psi(x, E, \epsilon)
\end{array}\right) \in \mathbb{C}^{2 m} .
$$

Then (3.1) is equivalent to

$$
i \epsilon^{2} \frac{\partial}{\partial x} \Phi(x, E, \epsilon)=H(x, E) \Phi(x, E, \epsilon),
$$

where

$$
\begin{aligned}
& H(x, E)=\left(\begin{array}{cc}
\mathscr{D} & I I \\
2(E I I-h(x)) & \mathscr{D}
\end{array}\right) \in M_{2 m}(\mathbb{C}) \quad \text { and } \\
& E I I-h(x)>0, \text { for all } x \in \mathbb{R} \cup\{ \pm \infty\} .
\end{aligned}
$$

Here, $I I$ denotes the identity matrix in $\mathbb{C}^{m}$. Note that the matrix $H(x, E)$ is not self-adjoint, but satisfies the relation

$$
H(x, E)=J H^{*}(x, E) J, \quad \text { where } \quad J=\left(\begin{array}{ll}
\text { D } & I I \\
I & \mathscr{D}
\end{array}\right) .
$$

The small $\epsilon$ asymptotics of solutions to (3.2) are studied in [15. Of particular importance to us is Section 7 of [15, which is devoted to the computation of exponentially small elements of the related $S$-matrix that we describe below. We apply the results of [15] to (3.2), keeping track of the dependence on $E$.

By our hypotheses on $E$ and $h(x)$, the spectrum of $H(E, x)$ consists of $2 m$ distinct real eigenvalues

$$
\begin{aligned}
& \sigma(H(x, E))=\left\{k_{j}^{\tau}(x, E)\right\}_{j=1, \cdots, m}^{\tau=+,-}, \quad \text { with } \\
& k_{j}^{\tau}(x, E)=\tau k_{j}(x, E)=\tau \sqrt{2\left(E-e_{j}(x)\right)} \in \mathbb{R} .
\end{aligned}
$$

Note that the $k_{j}^{\tau}$ 's correspond to the classical momenta associated with the classical potentials $e_{j}(x)$.

A set of corresponding eigenvectors $\left\{\chi_{j}^{\tau}(x, E)\right\}$ is given (in block notation) by

$$
\chi_{j}^{\tau}(x, E)=\left(\begin{array}{c}
\phi_{j}(x) \\
k_{j}^{\tau}(x, E) \phi_{j}(x)
\end{array}\right) \in \mathbb{C}^{2 m} .
$$

From these we produce new eigenvectors

$$
\varphi_{j}^{\tau}(x, E)=\chi_{j}^{\tau}(x, E) \frac{1}{\sqrt{2 k_{j}(x, E)}}
$$

that satisfy the normalization convention (3.10) below, that wasadopted in [15]. This normalization is motivated by the following: We can write

$$
H(x, E)=\sum_{j, \tau} k_{j}^{\tau}(x, E) P_{j}^{\tau}(x, E),
$$


where $\left\{P_{j}^{\tau}(x, E)\right\}$ denotes a set of non-orthogonal projections onto the eigenspaces of $H(x, E)$. If we define

$$
\theta_{j}^{\tau}(x, E)=\frac{1}{2}\left(\begin{array}{c}
\phi_{j}(x) \\
\frac{\tau}{k_{j}(x, E)} \phi_{j}(x)
\end{array}\right)
$$

then it is easy to check that

$$
P_{j}^{\tau}(x, E)=\left|\chi_{j}^{\tau}(x, E)\right\rangle\left\langle\theta_{j}^{\tau}(x, E)\right|,
$$

where we have used the bra-ket notation relative to the scalar product in $\mathbb{C}^{2 m}$. We use the same notation for scalar products in $\mathbb{C}^{m}$ and $\mathbb{C}^{2 m}$, since no confusion should arise.

We now see that the eigenvectors (3.7) satisfy the normalization conditions

$$
\left\{\begin{array}{cr}
P_{j}^{\tau}(x, E) \frac{\partial}{\partial x} \varphi_{j}^{\tau}(x, E) \equiv 0, & \text { and } \\
\left\langle\varphi_{j}^{\tau}(0, E), J \varphi_{j}^{\tau}(0, E)\right\rangle \equiv \tau \in\{-1,1\}
\end{array}\right.
$$

We note that $H, k_{j}^{\tau}, \chi_{j}^{\tau}, P_{j}^{\tau}$, and $\varphi_{j}^{\tau}$ are analytic functions of $x$ and $E$ when these variables are in a neighborhood of $\mathbb{R} \times \Delta$. More precisely, if $\Delta=\left[E_{1}, E_{2}\right]$, we define $D_{\beta}=\{z \in \mathbb{C}: \operatorname{dist}(z, \Delta)<\beta\}$, and these functions are analytic in $\rho_{\alpha} \times D_{\beta}$, for $\alpha$ and $\beta$ small enough. Here $\alpha$ must be chosen small enough so that $e_{j}$ and $\phi_{j}$ are analytic in $\rho_{\alpha}$, (see [20]), and $\beta$ must be small enough so that $\left|E-e_{j}(x)\right|>0$ in $\rho_{\alpha} \times D_{\beta}$.

We later make use of larger values of $\alpha$ in order to take advantage of the generic multivaluedness of $e_{j}$ and $\phi_{j}$ as functions of $x$.

From [15], we now see that any solution to (3.2) can be written as

$$
\Phi(x, E, \epsilon)=\sum_{j, \tau} c_{j}^{\tau}(x, E, \epsilon) e^{-i \int_{0}^{x} k_{j}^{\tau}(y, E) d y / \epsilon^{2}} \varphi_{j}^{\tau}(x, E),
$$

where the scalar coefficients $c_{j}^{\tau} \in \mathbb{C}$ satisfy the equation

$$
\frac{\partial}{\partial x} c_{j}^{\tau}(x, E, \epsilon)=\sum_{l, \sigma} a_{j l}^{\tau \sigma}(x, E) e^{i \int_{0}^{x}\left(\tau k_{j}(y, E)-\sigma k_{l}(y, E)\right) d y / \epsilon^{2}} c_{l}^{\sigma}(x, E, \epsilon),
$$

with

$$
a_{j l}^{\tau \sigma}(x, E)=-\frac{\left\langle\varphi_{j}^{\tau}(x, E), P_{j}^{\tau}(x, E) \frac{\partial}{\partial x} \varphi_{l}^{\sigma}(x, E)\right\rangle}{\left\|\varphi_{j}^{\tau}(x, E)\right\|^{2}} .
$$

We can rewrite (3.12) as an integral equation

$$
\begin{aligned}
c_{j}^{\tau}(x, E, \epsilon)= & c_{j}^{\tau}\left(x_{0}, E, \epsilon\right) \\
& +\int_{x_{0}}^{x} \sum_{l, \sigma} a_{j l}^{\tau \sigma}\left(x^{\prime}, E\right) e^{i \int_{0}^{x^{\prime}}\left(\tau k_{j}(y, E)-\sigma k_{l}(y, E)\right) d y / \epsilon^{2}} c_{l}^{\sigma}\left(x^{\prime}, E, \epsilon\right) d x^{\prime} .
\end{aligned}
$$


As we shall soon see, our hypotheses imply the existence of the $\operatorname{limits} \lim _{x \pm \infty} c_{j}^{\tau}(x, E, \epsilon)=$ $c_{j}^{\tau}( \pm \infty, E, \epsilon)$, so that with the notation

$$
\mathbf{c}^{\tau}(x, E, \epsilon)=\left(\begin{array}{c}
c_{1}^{\tau}(x, E, \epsilon) \\
c_{2}^{\tau}(x, E, \epsilon) \\
\vdots \\
c_{m}^{\tau}(x, E, \epsilon)
\end{array}\right) \in \mathbb{C}^{m}
$$

we can define an associated $S$-matrix, $\mathcal{S} \in M_{2 m}(\mathbb{C})$, by the identity

$$
\mathcal{S}(E, \epsilon)\left(\begin{array}{l}
\mathbf{c}^{+}(-\infty, E, \epsilon) \\
\mathbf{c}^{-}(-\infty, E, \epsilon)
\end{array}\right)=\left(\begin{array}{l}
\mathbf{c}^{+}(+\infty, E, \epsilon) \\
\mathbf{c}^{-}(+\infty, E, \epsilon)
\end{array}\right) .
$$

This $S$-matrix naturally takes the block form

$$
\mathcal{S}(E, \epsilon)=\left(\begin{array}{ll}
\mathcal{S}^{++}(E, \epsilon) & \mathcal{S}^{+-}(E, \epsilon) \\
\mathcal{S}^{-+}(E, \epsilon) & \mathcal{S}^{--}(E, \epsilon)
\end{array}\right) .
$$

Due to the symmetry (3.4), it also satisfies the relation (see [15]),

$$
\mathcal{S}^{-1}(E, \epsilon)=R \mathcal{S}^{*}(E, \epsilon) R, \quad \text { where } \quad R=\left(\begin{array}{cc}
I & \mathscr{D} \\
\mathscr{D} & -I
\end{array}\right) .
$$

Its elements describe transmission and reflection coefficients at fixed energy $E$ which play key roles in our analysis. The off-diagonal elements are exponentially small and their asymptotics are determined in [15].

With this notation, the generalized eigenvectors are given by

$$
\begin{aligned}
\Psi(x, E, \epsilon)=\sum_{j} & \frac{1}{\sqrt{2 k_{j}(x, E)}} \phi_{j}(x) \\
& \times\left(c_{j}^{+}(x, E, \epsilon) e^{-i \int_{0}^{x} k_{j}(y, E) d y / \epsilon^{2}}+c_{j}^{-}(x, E, \epsilon) e^{i \int_{0}^{x} k_{j}(y, E) d y / \epsilon^{2}}\right) .
\end{aligned}
$$

Since $\int_{0}^{x} k_{j}(y, E) d y \simeq x k_{j}( \pm \infty, E)=x \sqrt{2\left(E-e_{j}( \pm \infty)\right)}$ as $x \rightarrow \pm \infty$, the component of (3.16) that describes a wave traveling from the left to the right is labeled by -, and the component that describes a wave traveling from the right to the left is labeled by + . Note also that (3.16) is simply a WKB decomposition of the generalized eigenvectors.

We now state some of the general properties of the coefficients $c_{j}^{\tau}(x, E, \epsilon)$ and of the phases $e^{i \int_{0}^{x} k_{j}(y, E) d y / \epsilon^{2}}$ that allow us to justify the scattering results described above.

Lemma 3.1 Our hypotheses on $h(x)$ imply the following, uniformly for $E \in \Delta$ and all $n \in \mathbf{N}$ :

$$
\begin{aligned}
& 0<C_{1}(n) \leq \frac{\partial^{n}}{\partial E^{n}} k_{j}(x, E) \leq C_{2}(n)<\infty, \quad \text { and } \\
& \frac{\partial^{n}}{\partial E^{n}}\left(k_{j}^{\sigma}(x, E)-k_{j}^{\sigma}( \pm \infty, E)\right)=O\left(<x>^{-(2+\nu)}\right), \quad \text { as } \quad x \rightarrow \pm \infty .
\end{aligned}
$$


Thus, if we define $\omega_{j}^{\sigma}( \pm \infty, E)=\int_{0}^{ \pm \infty}\left(k_{j}^{\sigma}(y, E)-k_{j}^{\sigma}( \pm \infty, E)\right) d y$, we further have

$$
\int_{0}^{x} k_{j}^{\sigma}(y, E) d y=x k_{j}^{\sigma}( \pm \infty, E)+\omega_{j}^{\sigma}( \pm \infty, E)+r_{j}^{\sigma}( \pm, x, E)
$$

where, uniformly in $E$ and for all $n \in \mathbf{N}$,

$$
\frac{\partial^{n}}{\partial E^{n}} r_{j}^{\sigma}( \pm, x, E)=O\left(<x>^{-(1+\nu)}\right), \quad \text { as } \quad x \rightarrow \pm \infty .
$$

Moreover, the limits $c_{j}^{\sigma}( \pm \infty, E, \epsilon)$ as $x \rightarrow \pm \infty$ exist, and as $|x| \rightarrow \infty$,

$$
\frac{\partial^{n}}{\partial E^{n}} c_{j}^{\sigma}(x, E, \epsilon)=O(1), \quad \text { for } \quad n=0,1,
$$

uniformly for $E \in \Delta$. Also, as $x \rightarrow \pm \infty$ and uniformly for $E \in \Delta$,

$$
\begin{aligned}
& c_{j}^{\sigma}(x, E, \epsilon)-c_{j}^{\sigma}( \pm \infty, E, \epsilon)=O\left(<x>^{-(1+\nu)}\right), \quad \text { and } \\
& \frac{\partial}{\partial E}\left(c_{j}^{\sigma}(x, E, \epsilon)-c_{j}^{\sigma}( \pm \infty, E, \epsilon)\right)=O\left(<x>^{-\nu}\right) .
\end{aligned}
$$

\section{Remarks:}

1. This lemma is proved in Section 7

2. Some error terms in the lemma may depend on $\epsilon$ in a singular way as $\epsilon \rightarrow 0$. This will not matter because they will vanish in the scattering framework we adopt.

\subsection{Complex WKB Analysis}

All the information about transmissions and transitions among the asymptotic eigenstates of the electronic Hamiltonian is contained in the asymptotic values of the coefficients $c_{j}^{\sigma}(x, E, \pm \infty)$ defined by (3.13), and hence, in the matrix $\mathcal{S}(E, \epsilon)$. We extract this information by mimicking the complex WKB method of [15], while keeping track of the $E$ dependence.

The complex WKB method requires hypotheses on the behavior in the complex plane of the so-called Stokes lines for the equation (3.2) in order to provide the required asymptotics. These hypotheses are global in nature, and in general, are extremely difficult to check. However, in the physically interesting situation of "avoided crossings," they can be easily checked. We restrict our attention to these avoided crossing situations that are described below.

We consider the coefficients $c_{j}$ that are uniquely defined by the conditions

$$
c_{j}^{\tau}(-\infty, E, \epsilon)=1, \quad \text { and } \quad c_{k}^{\sigma}(-\infty, E, \epsilon)=0, \quad \text { for all } \quad(k, \sigma) \neq(j, \tau) .
$$

The key to the complex WKB method lies in the multivaluedness of the eigenvalues and eigenvectors of the analytic generator $H(x, E)$ of (3.2) in the complex $x$ plane. For any fixed $E \in \Delta, H(\cdot, E)$ is analytic in $\rho_{\alpha}$, and the solutions (3.11) to (3.2) are analytic in $x$ as well. 
However, the eigenvalues and eigenvectors may have branch points in $\rho_{\alpha}$ whose properties are inherited from those of the eigenvalues and eigenvectors of $h(\cdot)$.

Analytic perturbation theory as described in [20] states that the eigenvalues and eigenprojections of $h(x)$ for real $x$ are analytic on the real axis and admit analytic multivalued extensions to $\rho_{\alpha}$. The analytic continuations of the eigenvalues have branch points that are located on a set of crossing points

$$
\Omega=\left\{z_{0} \in \rho_{\alpha} \backslash \mathbb{R}: e_{j}\left(z_{0}\right)=e_{k}\left(z_{0}\right) \text { for some } j, k \text { and some analytic continuations }\right\} \text {. }
$$

Recall that for $\delta=0$, the eigenvalues are analytic at any crossing points on the real axis. This follows from the self-adjointness of $h(\cdot)$ on the real axis. Note also that $\bar{\Omega}=\Omega$ by the Schwarz reflection principle.

Generically, at a complex crossing point $z_{0} \in \Omega$, we have the following local behavior, where $c \in \mathbb{C}$ is some constant

$$
e_{j}(z)-e_{k}(z) \simeq c\left(z-z_{0}\right)^{1 / 2}\left(1+O\left(z-z_{0}\right)\right) .
$$

The eigenprojectors of $h(x)$ also admit multivalued extensions in $\rho_{\alpha} \backslash \Omega$, but they diverge at generic eigenvalue crossing points. We only have to deal with generic crossing points.

To see what happens to a multivalued function $f$ in $\rho_{\alpha} \backslash \Omega$ when we turn around a crossing point, we adopt the following convention: We denote by $f(z)$ the analytic continuation of $f$ defined in a neighborhood of the origin along some path from 0 to $z$. Then we perform the analytic continuation of $f(z)$ along a negatively oriented loop that surrounds only one point $z_{0} \in \Omega$. We denote by $\tilde{f}(z)$ the function we get by coming back to the original point $z$.

We define $\zeta_{0}$ to be a negatively oriented loop, based at the origin, that encircles only $z_{0}$ when $\operatorname{Im} z_{0}>0$. When $\operatorname{Im} z_{0}<0$, we choose $\zeta_{0}$ to be positively oriented.

We now fix $z_{0}$ with $\operatorname{Im} z_{0}>0$. If we analytically continue the set of eigenvalues $\left\{e_{j}(z)\right\}_{j=1}^{m}$, along a negatively oriented loop around $z_{0} \in \Omega$, we get the set $\left\{\widetilde{e}_{j}(z)\right\}_{j=1}^{m}$ with

$$
\tilde{e}_{j}(z)=e_{\pi_{0}(j)}(z), \quad \text { for } \quad j=1, \cdots, m,
$$

where

$$
\pi_{0}:\{1,2, \cdots, m\} \rightarrow\{1,2, \cdots, m\}
$$

is a permutation that depends on $z_{0}$. As a consequence, the eigenvectors (2.1) possess multivalued analytic extensions in $\rho_{\alpha} \backslash \Omega$. The analytic continuation $\widetilde{\phi}_{j}(z)$ of $\phi_{j}(z)$ along a negatively oriented loop around $z_{0} \in \Omega$, must be proportional to $\phi_{\pi_{0}(j)}(z)$. Thus, for $j=1,2, \cdots, m$, there exists $\theta_{j}\left(\zeta_{0}\right) \in \mathbb{C}$, such that

$$
\widetilde{\phi}_{j}(z)=e^{-i \theta_{j}\left(\zeta_{0}\right)} \phi_{\pi_{0}(j)}(z)
$$

We now turn from $h(x)$ to $H(x, E)$.

From Hypothesis H7, (3.5), and (3.7), we see that the set of crossing points for the eigenvalues $\pm k_{j}(x, E)$ of $H(x, E)$ is independent of $E$ and coincides with $\Omega$.

Moreover, for $j=1, \cdots, m$, we have

$$
\widetilde{k}_{j}^{\tau}(z, E)=k_{\pi_{0}(j)}^{\tau}(z, E), \quad \widetilde{\varphi}_{j}^{\tau}(z, E)=e^{-i \theta_{j}\left(\zeta_{0}\right)} \varphi_{\pi_{0}(j)}^{\tau}(z, E),
$$


where the prefactor $e^{-i \theta_{j}\left(\zeta_{0}\right)}$ is independent of $E$.

The above implies a key identity for the analytic extensions of the coefficients $c_{j}^{\tau}(z, E, \epsilon)$, $z \in \rho_{\alpha} \backslash \Omega$. Since the solutions to (3.2) are analytic for all $z \in \rho_{\alpha}$, the coefficients $c_{j}^{\tau}$ must also be multi-valued. In our setting, Lemma 3.1 of [15] implies the following lemma.

Lemma 3.2 For any $j=1, \cdots, m$ and $\tau=+,-$, we have

$$
\widetilde{c}_{j}^{\tau}(z, E, \epsilon) e^{-i \int_{\zeta_{0}} k_{j}^{\tau}(u, E) d u / \epsilon^{2}} e^{-i \theta_{j}\left(\zeta_{0}\right)}=c_{\pi_{0}(j)}^{\tau}(z, E, \epsilon)
$$

where $\zeta_{0}, \theta_{j}\left(\zeta_{0}\right)$ and $\pi_{0}(j)$ are defined as above and are independent of $E \in \Delta$.

Remark: Since $\Omega$ is finite, it is straightforward to generalize the study of the analytic continuations around one crossing point to analytic continuations around several crossing points. The loop $\zeta_{0}$ can be rewritten as a concatenation of finitely many individual loops, each encircling only one point of $\Omega$. The permutation $\pi_{0}$ is given by the composition of associated permutations. The factors $e^{-i \theta_{j}\left(\zeta_{0}\right)}$ in (3.21) are given by the product of the factors associated with the individual loops. The same is true for the factors $\exp \left(-i \int_{\zeta_{0}} k_{j}^{\tau}(z, E) d z / \epsilon^{2}\right)$ in Lemma 3.2 .

We now describe how we use the above properties. The details may be found in 15.

The idea is to integrate the integral equation corresponding to (3.13) along paths that go above (or below) one or several crossing points, and then to compare the result with the integration performed along the real axis. As $z \rightarrow-\infty$ in $\rho_{\alpha}$ these paths become parallel to the real axis so that the coefficients take the same asymptotic value $c_{m}^{\tau}(-\infty, E, \epsilon)$ along the real axis and the integration paths. Since the solutions to (3.2) are analytic, the results of these integrations must agree as $\operatorname{Re} z \rightarrow \infty$. Therefore, (3.22) taken at $z=\infty$ yields the asymptotics of $c_{\pi_{0}(j)}^{\tau}(\infty, E, \epsilon)$, provided we can control $\widetilde{c}_{j}(z, E, \epsilon)$ in the complex plane. We argue below that this can be done in the so-called dissipative domains (See [6], [5]), as proven in [15]. We do not go into the details of these notions because another result of [15] will enable us to get sufficicient control on $\widetilde{c}_{j}(z, E, \epsilon)$ in the avoided crossing situation, to which we restrict our attention.

We define

$$
\Delta_{j l}^{\tau \sigma}(x, E)=\int_{0}^{x}\left(k_{j}^{\tau}(y, E)-k_{j}^{\sigma}(y, E)\right) d y .
$$

By explicit computation, using formula (7.3) in (3.13), we check that (3.13) can be extended to $\rho_{\alpha} \backslash \Omega$. We next integrate by parts in (3.13), to see that (3.13) with $x_{0}=-\infty$ can be rewritten as

$$
\begin{aligned}
\widetilde{c}_{m}^{\tau}(z, E, \epsilon)= & \delta_{j m} \delta_{\tau-}-i \epsilon^{2} \sum_{l, \sigma} \frac{\widetilde{a}_{m l}^{\tau \sigma}(z, E)}{\widetilde{k}_{m}^{\tau}(z, E)-\widetilde{k}_{l}^{\sigma}(z, E)} e^{i \widetilde{\Delta}_{m l}^{\tau \sigma}(z, E) / \epsilon^{2}} \widetilde{c}_{l}^{\sigma}(z, E, \epsilon) \\
& +i \epsilon^{2} \sum_{l, \sigma} \int_{-\infty}^{z}\left(\frac{\partial}{\partial z^{\prime}} \frac{\widetilde{a}_{m l}^{\tau \sigma}\left(z^{\prime}, E\right)}{\widetilde{k}_{m}^{\tau}\left(z^{\prime}, E\right)-\widetilde{k}_{l}^{\sigma}\left(z^{\prime}, E\right)}\right) e^{i \widetilde{\Delta}_{m l}^{\tau \sigma}\left(z^{\prime}, E\right) / \epsilon^{2}} \widetilde{c}_{l}^{\sigma}\left(z^{\prime}, E, \epsilon\right) d z^{\prime} \\
& +i \epsilon^{2} \sum_{l, p, \sigma, \theta} \int_{-\infty}^{z} \frac{\widetilde{a}_{m l}^{\tau \sigma}\left(z^{\prime}, E\right) \widetilde{a}_{l p}^{\sigma \theta}\left(z^{\prime}, E\right)}{\widetilde{k}_{m}^{\tau}\left(z^{\prime}, E\right)-\widetilde{k}_{l}^{\sigma}\left(z^{\prime}, E\right)} e^{i \widetilde{\Delta}_{m p}^{\tau \theta}\left(z^{\prime}, E\right) / \epsilon^{2}} \widetilde{c}_{p}^{\theta}\left(z^{\prime}, E, \epsilon\right) d z^{\prime}
\end{aligned}
$$


as long as the chosen path of integration does not meet $\Omega$. Here, $\sim$ denotes the analytic continuation along the chosen path of integration of the corresponding function defined originally on the real axis. This distinguishes $\widetilde{c}_{m}^{\tau}(\infty, E, \epsilon)$ from $c_{m}^{\tau}(\infty, E, \epsilon)$ computed along the real axis as $x \rightarrow \infty$. These quantities may differ since the integration path may pass above (or below) points of $\Omega$.

If the exponentials in (3.23) are all uniformly bounded, as it is the case when the integration path coincides with the real axis, it is straighforward to get bounds of the type

$$
\tilde{c}_{m}^{\tau}(z, E, \epsilon)=\delta_{j m} \delta_{\tau-}+O_{E}\left(\epsilon^{2}\right) .
$$

In our context, all quantities depend on $E \in \Delta$. However, by mimicking the proof of Proposition 4.1 of 15], it is not difficult to check that the estimate (3.24) is uniform for $E \in \Delta$. For later purposes, we note that by differentiating (3.23), $\frac{\partial}{\partial E} \widetilde{c}_{m}^{\tau}(z, E, \epsilon)$ is uniformly bounded for $0<\epsilon<\epsilon_{0}$ and $E \in \Delta$ for any fixed $\epsilon_{0}$.

Again, as is well known, the existence of paths from $-\infty$ to $+\infty$ along which the exponentials do not blow up and which pass above (or below) points in $\Omega$ is difficult to check in general. It is linked to the global behavior of the Stokes lines of the problem. See e.g., [6], [5]. This property goes under the name "existence of dissipative domains" in [15].

We avoid these complications by restricting attention to avoided crossing situations where the existence has been proven [15].

When dissipative domains exist, (3.22) and (3.24) imply

$$
c_{\pi_{0}(j)}^{\tau}(\infty, E, \epsilon)=e^{-i \int_{\zeta_{0}} k_{j}^{\tau}(u, E) d u / \epsilon^{2}} e^{-i \theta_{j}\left(\zeta_{0}\right)}\left(1+O_{E}\left(\epsilon^{2}\right)\right),
$$

where the $O_{E}\left(\epsilon^{2}\right)$ estimate is uniform for $E \in \Delta$. This is the main result of Proposition 4.1 in [15] in our context, under the assumption that dissipative domains exist.

\subsection{Avoided Crossings}

We now explore the avoided crossing situation, alluded to above, that allows us to avoid considerations of the dissipative domains. We now assume that $h(x)$ has the form $h(x, \delta)$ and satisfies Hypotheses $\mathbf{H} 4$ and $\mathbf{H 5 .}$

We first check that the allowed pattern of avoided crossings for $\sigma(h(x, \delta))$ can be transfered to the eigenvalues of $H(x, E, \delta)$, obtained from $h(x, \delta)$ by (3.3).

From the explicit formulae (3.5), we see immediately that $x_{c} \in \mathbb{R}$ is a real crossing point for the eigenvalues $e_{j}(x, 0)$ and $e_{l}(x, 0)$ of $h(x, 0)$ if and only if it is a real crossing point for the analytic eigenvalues $k_{j}^{\tau}(x, E, 0)$ and $k_{l}^{\tau}(x, E, 0)$ of $H(x, E, 0)$, for $\tau=+,-$. Moreover,

$$
\left.\frac{\partial}{\partial x}\left(k_{j}^{\tau}(x, E)-k_{l}^{\tau}(x, E)\right)\right|_{x=x_{c}}=\left.\tau \frac{\frac{\partial}{\partial x}\left(e_{l}(x, 0)-e_{j}(x, 0)\right)}{\sqrt{2\left(E-e_{j}(x, 0)\right)}}\right|_{x=x_{c}},
$$

so that the real crossings for $H(x, E, 0)$ are also generic, in the sense of (2.5). 
Remark: Our assumption $\mathbf{H 7}$ on the parameter $E$ forbids real crossings between eigenvalues $k_{j}^{\tau}(x, E, 0)$ and $k_{l}^{\sigma}(x, E, 0)$, with $\sigma \neq \tau$.

Regarding the ordering of the eigenvalues of $H(x, E, \delta)$, if those of $h(x, \delta)$ are ordered as in (2.4), we have

$$
-k_{1}(x, E, \delta)<\cdots<-k_{m}(x, E, \delta)<0<k_{m}(x, E, \delta)<\cdots<k_{1}(x, E, \delta) .
$$

This means that the pattern of the crossings for the group of eigenvalues $\left\{-k_{j}(x, E, 0)\right\}_{j=1, \cdots, m}$ is the same as that for the eigenvalues $\left\{e_{j}(x, 0)\right\}_{j=1, \cdots, m}$. The pattern of the crossings for the group $\left\{k_{j}(x, E, 0)\right\}_{j=1, \cdots, m}$ is the reflection with respect to the horizontal axis of the one for $\left\{e_{j}(x, 0)\right\}_{j=1, \cdots, m}$. Therefore, assumptions $\left.\mathbf{H 5}, \mathrm{i}\right)$, ii), iii) are also satisfied for the eigenvalues of $H(x, E, 0)$, for a relabeling from 1 to $2 m$ of (3.26) with $\delta=0$, and $x$ close to $-\infty$.

To any given pattern of real crossings for the eigenvalues $\left\{e_{j}(x, 0)\right\}_{j=1, \cdots, m}$ of $h(x, 0)$, we associate a permutation $\pi$ of $\{1,2, \cdots, m\}$ as follows. Assume the eigenvalues are labeled in ascending order at $x=-\infty$, as in property i) of $\mathbf{H 5}$. If $e_{j}(\infty, 0)$ is the $k^{\text {th }}$ eigenvalue in ascending order at $x=\infty$, the permutation $\pi$ is defined by

$$
\pi(j)=k .
$$

We call $\pi$ the permutation associated with $\sigma(h(x, 0))$. For small $\delta>0$, the real crossings turn into avoided crossings on the real axis and conjugate complex crossing points appear close to the real axis. Then $\pi$ corresponds to the permutation $\pi_{0}$ (3.20) for a loop $\zeta_{0}$ that surrounds all complex crossing points in the upper half plane that are associated with the avoided crossings.

These properties of corresponding patterns of real crossings of the spectra of $h(x, \delta)$ and $H(x, E, 0)$ immediately yield the following convenient relation between the permutation $\pi$ associated with $\sigma(h(x, 0))$ and the permutation $\Pi$ associated with $\sigma(H(x, E, 0))$. If we denote $\Pi$ by the obvious notation

$$
\Pi(j, \tau)=(k, \sigma)
$$

then we have

$$
\Pi(j, \tau)=(\pi(j), \tau), \quad \text { for all } \quad(j, \tau) \in\{1, \cdots, m\} \times\{-,+\}
$$

We can now restate the main result of [15] that describes the asymptotics of the coefficients defined in (3.13), adapted to our scattering framework for incoming states entering from the left. (See (3.16).) Intuitively, this result says that for small $\delta>0$, dissipative domains exist, provided the pattern of real crossings satisfies H5. Therefore, estimates of the type (3.25) are true for certain indices $j$ and $n$, determined by the permutation (3.27). It is not difficult to see that the permutation $\pi$ describes the successive exchanges of eigenvalues one gets by following a path in the complex plane that goes above or below all complex crossing points of the eigenvalues $e_{j}(x, \delta)$ that are associated with the avoided crossings. 
Theorem 3.1 Let $h(x, \delta)$ satisfy $\mathbf{H 4}$ and $\mathbf{H 5}$. If $\delta>0$ is small enough, the $\pi(j), j$ elements of the matrix $\mathcal{S}^{--}(E, \epsilon)$ in (3.15), with $\pi(j)$ defined in (3.27), have small $\epsilon$ asymptotics for all $j=1, \cdots, m$, given by

$$
\mathcal{S}_{\pi(j), j}^{--}(E, \epsilon)=\prod_{l=j}^{\pi(j) \mp 1} e^{-i \theta_{l}\left(\zeta_{l}, \delta\right)} e^{i \int_{\zeta_{l}} k_{l}(z, E, \delta) d z / \epsilon^{2}}\left(1+O_{E, \delta}\left(\epsilon^{2}\right)\right), \quad \pi(j)\left\{\begin{array}{l}
>j \\
<j
\end{array}\right.
$$

where, for $\pi(j)>j$ (respectively $\pi(j)<j), \zeta_{l}, l=j, \cdots, \pi(j)-1$ (resp. $\left.l=j, \cdots, \pi(j)+1\right)$, denotes a negatively (resp. positively) oriented loop based at the origin which encircles the complex crossing point $z_{r}$ only (resp. $\overline{z_{r}}$ ) corresponding to the avoided crossing between $e_{l}(x, \delta)$ and $e_{l+1}(x, \delta)$ (resp. $\left.e_{l-1}(x, \delta)\right)$ at $x_{r}$. The $\int_{\zeta_{l}} k_{l}(z, E, \delta) d z$ denotes the integral along $\zeta_{l}$ of the analytic continuation of $k_{l}(0, E, \delta)$, and $\theta_{l}\left(\zeta_{l}\right)$ is the corresponding factor defined by (3.21).

\section{Remarks:}

i) Revisiting the proof of this theorem in [15], we see that we can choose $\delta>0$ small enough so that dissipative domains can be constructed uniformly for $E \in \Delta$. This stems from the formula

$$
k_{j}(x, E, 0)-k_{l}(x, E, 0)=\frac{2\left(e_{l}(x, 0)-e_{j}(x, 0)\right)}{k_{j}(x, E, 0)+k_{l}(x, E, 0)},
$$

whose denominator can be controlled, close to the real axis, uniformly for $E \in \Delta$.

ii) When there is only one avoided crossing between level $j$ and $j+1$ stemming from a real crossing at $x=x_{0}$, we have $j+1=\pi(j)$. The theorem says

$$
\mathcal{S}_{(j+1), j}^{--}(E, \epsilon)=e^{-i \theta_{j}\left(\zeta_{j}, \delta\right)} e^{i \int_{\zeta_{j}} k_{j}(z, E, \delta) d z / \epsilon^{2}}\left(1+O_{E, \delta}\left(\epsilon^{2}\right)\right),
$$

where the negatively oriented loop $\zeta_{j}$ encircles the corresponding complex crossing point $z_{0}$, with $\operatorname{Im} z_{0}>0$. Similarly, interchanging the roles of $j$ and $j+1$, it yields with $\bar{\zeta}_{j}$ the conjugate of the loop $\zeta_{j}$,

$$
\mathcal{S}_{j(j+1)}^{--}(E, \epsilon)=e^{-i \theta_{j}\left(\bar{\zeta}_{j}, \delta\right)} e^{i \int_{\bar{\zeta}_{j}} k_{j}(z, E, \delta) d z / \epsilon^{2}}\left(1+O_{E, \delta}\left(\epsilon^{2}\right)\right)
$$

iii) Since the eigenvalues are continuous at the complex crossings, we have

$$
\lim _{\delta \rightarrow 0} \operatorname{Im} \int_{\zeta_{j}} k_{j}(z, E, \delta) d z=0, \quad \text { for all } \quad j=1, \cdots, p .
$$

It is shown in 14 that

$$
\lim _{\delta \rightarrow 0} \operatorname{Im} \theta_{j}\left(\zeta_{j}, \delta\right)=0 \quad \text { for all } \quad j=1, \cdots, p
$$

iv) The $O_{E, \delta}(\epsilon)$ errors in Theorem 3.1 depend on $\delta$, but it should be possible to get estimates which are valid as both $\epsilon$ and $\delta$ tend to zero, in the spirit of [14, 21], and [25].

v) This result shows that at least one off-diagonal element per column of the $S$-matrix can be computed asymptotically. However, it is often possible to get more elements by making 
use of symmetries of the $S$-matrix. See [15] and [16].

In our avoided crossings context, transitions of the coefficients between states that correspond to electronic levels that do not display avoided crossings, i.e., that are separated by a gap of order 1 as $\delta \rightarrow 0$, are expected to be exponentially smaller than the transitions we control by means of Theorem 3.1, as $\delta$ shrinks to zero. Since the coefficients in the exponential decay rates given by the theorem vanish in the limit $\delta \rightarrow 0$, it is enough to show that the decay rates of the exponentially small transitions between well separated levels are independent of $\delta$.

That is the meaning of the following proposition, which draws heavily upon [18] and [15] and is proven in Section 7

Proposition 3.1 Let $F(x, \delta)$ be a $n \times n$ matrix that satisfies $\mathbf{H 4}$, except for the condition that $F(\cdot, \delta)$ be self-adjoint. Suppose its eigenvalues $\left\{f_{j}(x, \delta)\right\}_{j=1, \cdots, m}$ that satisfy $\mathbf{H 5}$. Further assume that the eigenvalues can be separated into two groups $\sigma_{1}(x, \delta)$ and $\sigma_{2}(x, \delta)$ that display no avoided crossing, i.e., such that

$$
\inf _{\substack{\delta \geq 0 \\ x \in \rho_{\alpha} \cup\{ \pm \infty\}}} \operatorname{dist}\left(\sigma_{1}(x, \delta), \sigma_{2}(x, \delta)\right) \geq g>0 .
$$

Let $P(x, \delta)$ and $Q(x, \delta)=\Pi-P(x, \delta)$ be the projectors onto the spectral subspaces corresponding to $\sigma_{1}(x, \delta)$ and $\sigma_{2}(x, \delta)$ respectively, and let $U_{\epsilon}\left(x, x_{0}, \delta\right)$ be the evolution operator corresponding to the equation

$$
i \epsilon^{2} \frac{d}{d x} U_{\epsilon}\left(x, x_{0}, \delta\right)=F(x, \delta) U_{\epsilon}\left(x, x_{0}, \delta\right), \quad \text { with } \quad U_{\epsilon}\left(x_{0}, x_{0}, \delta\right)=I I .
$$

Then, for any $\delta>0$, there exists $\epsilon_{0}(\delta), C(\delta)>0$ depending on $\delta$, and $\Gamma>0$ independent of $\delta$, such that for all $\epsilon \leq \epsilon_{0}(\delta)$,

$$
\lim _{\substack{x \rightarrow \infty \\ x_{0} \rightarrow-\infty}}\left\|P(x, \delta) U_{\epsilon}\left(x, x_{0}, \delta\right) Q\left(x_{0}, \delta\right)\right\| \leq C(\delta) e^{-\Gamma / \epsilon^{2}}
$$

Remark: This proposition implies that reflections, i.e., the transitions from wave packets traveling to the right to wave packets traveling to the left, on any electronic level, are exponentially smaller than transitions associated with the avoided crossings in which the propagation direction is not changed. This is a consequence of Hypothesis $\mathbf{H} \mathbf{7}$ which implies that complex crossings between $k_{j}^{+}$and $k_{l}^{-}$, are far from the real axis for any $j, l \in\{1, \cdots, m\}$.

Let us investigate more closely the analytic structure of $k_{j}(z, E, \delta)$ in our avoided crossing regime characterized by $\mathbf{H} \mathbf{4}$ and $\mathbf{H 5}$, in order to deduce the properties of the exponential decay rates $\operatorname{Im} \int_{\zeta_{j}} k_{j}(z, E, \delta) d z$. We do so for the $k_{j}$ 's that correspond to electronic eigenvalues $e_{j}(z, \delta)$ and $e_{n}(z, \delta)$ that experience only one avoided crossing, i.e., we take $n=j \pm 1$. We can thus drop the index $j$ in $\zeta_{j}$ in the notation. We follow 14 where a similar analysis is performed, sometimes refering to results proven there. The general case is dealt with by adding the corresponding contributions stemming from each individual avoided crossing. 
We can assume that the avoided crossing takes place at $x=0$, i.e.,

$$
e_{j}(0,0)=e_{n}(0,0) \equiv e_{c}
$$

where $e_{c}$ is the electronic eigenvalue at the crossing. We also define the momentum $k_{c}(E)$ at the crossing point by

$$
k_{c}(E)=\sqrt{2\left(E-e_{c}\right)}
$$

and the quantity $\Gamma_{0}(\delta)$ by

$$
\Gamma_{0}(\delta)=\left|\operatorname{Im} \int_{\zeta} e_{j}(z, \delta) d z\right|
$$

This quantity is the exponential decay rate given by the Landau-Zener Formula for a (time dependent) adiabatic problem with hamiltonian $h(t, \delta)$. See [14]. In Section 7 we prove

Lemma 3.3 With the above notation, we have the following as $\delta \rightarrow 0$, uniformly for $E \in \Delta$,

$$
\begin{aligned}
& \operatorname{Im} \int_{\zeta} \sqrt{2\left(E-e_{j}(z, \delta)\right)} d z=\frac{\Gamma_{0}(\delta)}{k_{c}(E)}+O\left(\delta^{3}\right) \\
& \frac{\partial}{\partial E} \operatorname{Im} \int_{\zeta} \sqrt{2\left(E-e_{j}(z, \delta)\right)} d z=-\frac{\Gamma_{0}(\delta)}{k_{c}^{3}(E)}+O\left(\delta^{3}\right), \quad \text { and } \\
& \frac{\partial^{2}}{\partial E^{2}} \operatorname{Im} \int_{\zeta} \sqrt{2\left(E-e_{j}(z, \delta)\right)} d z=3 \frac{\Gamma_{0}(\delta)}{k_{c}^{5}(E)}+O\left(\delta^{3}\right),
\end{aligned}
$$

where $0<\Gamma_{0}(\delta)=O\left(\delta^{2}\right)$.

\section{Remarks:}

i) This implies that $\operatorname{Im} \int_{\zeta} \sqrt{2\left(E-e_{j}(z, \delta)\right)} d z$ is a positive, decreasing, convex function of $E$ on $\Delta$. This remains true when the transition is mediated by several avoided crossings.

ii) The first relation can be interpreted as saying that in our Born-Oppenheimer context, the (time dependent adiabatic) Landau-Zener decay rate at fixed energy $E$ has to be modified in order to take into account the classical velocity $k_{c}(E)$ at the crossing.

iii) More precise estimates will be derived below, further assuming H6.

\section{Exact Solutions to the Time-Dependent Schrödinger Equation}

We now construct solutions to (1.1) by taking time-dependent superpositions of the generalized eigenvectors $\Psi(x, E, \epsilon)$, where $E \in \Delta$. These superpositions depend on an energy density $Q(E, \epsilon)$ that can be complex and may or may not depend on $\epsilon$. We always assume that the following condition holds:

C0 : The density $Q(E, \epsilon)$ is $C^{1}$ as a function of $E \in \Delta$, for fixed $\epsilon>0$. 
In this Section, the parameter $\delta>0$ is kept fixed and plays no role. We therefore drop it from the notation and work under hypotheses $\mathbf{H 1}, \mathbf{H} \mathbf{2}$, and $\mathbf{H 3}$.

We define

$$
\begin{aligned}
\psi(x, t, \epsilon) & =\int_{\Delta} Q(E, \epsilon) \Psi(x, E, \epsilon) e^{-i t E / \epsilon^{2}} d E \\
& =\sum_{j=1, \cdots, m, \sigma= \pm} \phi_{j}(x) \int_{\Delta} \frac{Q(E, \epsilon)}{\sqrt{2 k_{j}(x, E)}} c_{j}^{\sigma}(x, E, \epsilon) e^{-i \int_{0}^{x} k_{j}^{\sigma}(y, E) d y / \epsilon^{2}} e^{-i t E / \epsilon^{2}} d E \\
& \equiv \sum_{j=1, \cdots, m} \psi_{j= \pm}^{\sigma}(x, t, \epsilon) .
\end{aligned}
$$

Here $\psi_{j}^{\sigma}$ asymptotically describes the piece of the solution that lives on the electronic state $\phi_{j}$ and propagates in the direction characterized by $\sigma$. Since the integrand is smooth and $\Delta$ is compact, $\psi(x, t, \epsilon)$ is an exact solution to the time-dependent Schrödinger equation (1.1). Note that this solution is not necessarily normalized.

The following lemma, whose proof can be found in Section 17 gives a bound that we use to understand the large $t$ behavior of $\psi_{j}^{\sigma}(x, t, \epsilon)$. It is a simple corollary that the state (4.1) belongs to $L^{2}(\mathbb{R})$.

Lemma 4.1 Assume H1, H2, H3 and C0. Let

$$
K_{+}=\sup _{j=1, \cdots, m} k_{E \in \Delta, \sigma= \pm}(\sigma \infty, E)<\infty
$$

and

$$
K_{-}=\inf _{j=1, \cdots, m} k_{E \in \Delta, \sigma= \pm}(\sigma \infty, E)>0 .
$$

Fix $\alpha \in(0,1)$. Then, for either $t=0$, or for any $x \neq 0$ and $t \neq 0$, such that

$$
|x / t|>K_{+} /(1-\alpha) \quad \text { or } \quad|x / t|<K_{-} /(1+\alpha),
$$

we have

$$
\left\|\psi_{j}^{\sigma}(x, t, \epsilon)\right\| \leq C_{\epsilon} /|x|, \quad \text { with } C_{\epsilon} \text { independent of } t,
$$

where the estimate is in the norm on $\mathbb{C}^{m}$.

We now introduce freely propagating states $\psi(x, t, \epsilon, \pm) \in L^{2}\left(\mathbb{R}, \mathbb{C}^{m}\right)$ that describe the asymptotics of the solutions $\psi(x, t, \epsilon)$ as $t \rightarrow \pm \infty$. We use these asymptotic states when we study the scattering matrix for (1.1). We let

$$
\begin{aligned}
& \psi(x, t, \epsilon, \pm) \\
& =\sum_{j=1, \cdots, m} \phi_{j= \pm}(x) \int_{\Delta} \frac{Q(E, \epsilon)}{\sqrt{2 k_{j}( \pm \infty, E)}} e^{-i t E / \epsilon^{2}} c_{j}^{\sigma}( \pm \infty, E, \epsilon) e^{-i\left(x k_{j}^{\sigma}( \pm \infty, E)+\omega_{j}^{\sigma}( \pm \infty, E)\right) / \epsilon^{2}} d E \\
& =\sum_{j=1, \cdots, m} \psi_{j= \pm}^{\sigma}(x, t, \epsilon, \pm)
\end{aligned}
$$


These states are linear combinations of products of free scalar wave packets in constant scalar potentials times eigenvectors of the electronic Hamiltonian. Their propagation is thus governed by the various channel Hamiltonians.

Proposition 4.1 Assume H1, H2, H3 and C0. In $L^{2}(\mathbb{R})$ norm as $t \rightarrow \pm \infty$, we have

$$
\|\psi(x, t, \epsilon)-\psi(x, t, \epsilon, \pm)\|=O_{\epsilon}(1 /|t|) .
$$

\section{Remarks:}

i) The estimate (4.3) depends on $\epsilon$.

ii) By a change of variables, we immediately obtain the following corollary.

Corollary 4.1 The density of the component of the asymptotic momentum space wave function on the $j^{\text {th }}$ electronic level as $t \rightarrow \pm \infty$ is

$$
\sigma \sqrt{\frac{k}{2}} Q(E(k), \epsilon) c_{j}^{\sigma}( \pm \infty, E(k), \epsilon) e^{-i \omega_{j}^{\sigma}( \pm \infty, E(k)) / \epsilon^{2}}
$$

Here $E(k)=k^{2} / 2+e_{j}( \pm \infty)$ and $\sigma=-/+$ for waves traveling in the positive/negative direction, respectively.

iii) Consider a solution $\psi(x, t, \epsilon)$ traveling in the positive direction and associated with the electronic eigenstate $\phi_{j}$ in the remote past. It is characterized by $c_{k}^{\sigma}(-\infty, E, \epsilon)=\delta_{k, j} \delta_{\sigma,-}$, and as $t \rightarrow-\infty$, it is asymptotic to

$$
\psi(x, t, \epsilon,-)=\phi_{j}(x) \int_{\Delta} \frac{Q(E, \epsilon)}{\sqrt{2 k_{j}(-\infty, E)}} e^{-i t E / \epsilon^{2}} e^{i\left(x k_{j}(-\infty, E)-\omega_{j}^{-}(-\infty, E)\right) / \epsilon^{2}} d E .
$$

As $t \rightarrow+\infty$, the component of this state that has made the transition from state $j$ to state $n$ is asymptotic to the vector $\psi_{n}^{-}(x, t, \epsilon,+)$. It is given in terms of the matrix $\mathcal{S}$ by

$$
\phi_{n}(x) \int_{\Delta} \frac{Q(E, \epsilon)}{\sqrt{2 k_{n}(+\infty, E)}} e^{-i t E / \epsilon^{2}} \mathcal{S}_{n j}^{--}(E, \epsilon) e^{+i\left(x k_{n}(+\infty, E)-\omega_{n}^{-}(+\infty, E)\right) / \epsilon^{2}} d E .
$$

iv) Proposition 4.1 is proven in the Section 7.

\section{Non-adiabatic Transition Asymptotics}

\subsection{The Transition Integral}

From now on, we assume we are in the avoided crossing situation, but we still do not make explicit the dependence in the variable $\delta>0$ in the notation.

Section 3 gave us the semiclassical asymptotics of the elements of the $S$-matrix $\mathcal{S}(E, \epsilon)$. We now compute the small $\epsilon$ asymptotics of the integrals that describe the asymptotic states $\psi_{j}^{\sigma}(x, t, \epsilon, \pm)$ given by (4.2) as $|t| \rightarrow \infty$, for the different channels. 
We choose our energy density $Q(E, \epsilon)$ to be more and more sharply peaked near a specific value $E_{0} \in \Delta \backslash \partial \Delta$ as $\epsilon \rightarrow 0$. As a result, we obtain semiclassical Born-Oppenheimer states that are well localized in phase space. This choice is physically reasonable, and it allows us to relate the quantum scattering process to classical quantities.

More precisely we consider,

$$
Q(E, \epsilon)=e^{-G(E) / \epsilon^{2}} e^{-i J(E) / \epsilon^{2}} P(E, \epsilon),
$$

where

C1 : The real-valued function $G \geq 0$ is in $C^{3}(\Delta)$, and has a unique non-degenerate absolute minimum value of 0 at $E_{0}$ in the interior of $\Delta$. This implies that

$$
G(E)=g\left(E-E_{0}\right)^{2} / 2+O\left(E-E_{0}\right)^{3}, \quad \text { where } \quad g>0 .
$$

C2 : The real-valued function $J$ is in $C^{3}(\Delta)$.

C3 : The complex-valued function $P(E, \epsilon)$ is in $C^{1}(\Delta)$ and satisfies

$$
\sup _{\substack{E \in \Delta \\ \epsilon \geq 0}}\left|\frac{\partial^{n}}{\partial E^{n}} P(E, \epsilon)\right| \leq C_{n}, \quad \text { for } \quad n=0,1 .
$$

Remark: Typical interesting choices of $Q$ have $G=g\left(E-E_{0}\right)^{2}, J=0$, and $P$ an $\epsilon$-dependent multiple of a smooth function with at most polynomial growth in $\left(E-E_{0}\right) / \epsilon$.

In our avoided crossing situation, we have already proved the following: A wave packet incoming from the left in the remote past produces reflected waves (i.e., components that travel to the left in the remote future) that are exponentially smaller than the components that travel to the right in the remote future. We have also proved that the non-trivial transitions to electronic states that are not involved in the avoided crossing are exponentially smaller than those to electronic states that are involved in the avoided crossing.

Thus, the leading non-adiabatic transitions are described by the asymptotics of those coefficients $c_{l}^{\sigma}( \pm \infty, E, \epsilon)$ that satisfy

$$
\begin{aligned}
c_{k}^{\sigma}(-\infty, E, \epsilon) & =\delta_{j, k} \delta_{\sigma,-} \\
c_{n}^{-}(+\infty, E, \epsilon) & =e^{-i \theta_{j}(\zeta)} e^{i \int_{\zeta} k_{j}(z, E) d z / \epsilon^{2}}\left(1+O_{E}\left(\epsilon^{2}\right)\right),
\end{aligned}
$$

where $n=\pi(j)=j \pm 1$. We recall that the error term $O_{E}\left(\epsilon^{2}\right)$ depends analytically on the energy $E$ in a neighborhood of the compact set $\Delta$. We have already noted in the comments after (3.24) that the term $O_{E}\left(\epsilon^{2}\right)$ satisfies (15.2).

The form chosen for the energy densities should make it clear that Gaussian wave packets will play a particular role in the asymptotic analysis of (4.5). Therefore we use the specific notation introduced in (1.2) for them.

Recall that a normalized free Gaussian state propagating in the constant potential $e(+\infty)$ is characterized by the classical quantities

$$
A_{+}(t)=A_{+}+i t B_{+},
$$




$$
\begin{aligned}
B_{+}(t) & =B_{+}, \\
a_{+}(t) & =a_{+}+\eta_{+} t, \\
\eta_{+}(t) & =\eta_{+}, \\
S_{+}(t) & =\int_{0}^{t}\left(\eta_{+}^{2}(s) / 2-e(+\infty)\right) d s,
\end{aligned}
$$$$
\text { and }
$$

with $\operatorname{Re}\left(\overline{A_{+}} B_{+}\right)=1$ (see $\left.[9]\right)$. The associated nuclear wave packet has the form

$$
\begin{aligned}
& e^{i S_{+}(t) / \epsilon^{2}} \varphi_{0}\left(A_{+}(t), B_{+}(t), \epsilon^{2}, a_{+}(t), \eta_{+}(t), x\right) \\
& =\frac{e^{i t\left(\eta^{2} / 2-e(\infty)\right) / \epsilon^{2}}}{\pi^{1 / 4} \sqrt{\epsilon\left(A_{+}+i t B_{+}\right)}} \exp \left\{-\frac{\left(x-\left(a_{+}+\eta_{+} t\right)\right)^{2} B_{+}}{2 \epsilon^{2}\left(A_{+}+i t B_{+}\right)}\right\} \exp \left\{i \frac{\eta_{+}\left(x-\left(a_{+}+\eta_{+} t\right)\right)}{\epsilon^{2}}\right\} .
\end{aligned}
$$

We now have everything to state our main result:

Theorem 5.1 Let $\psi(x, t, \epsilon)$ be a solution of the Schrödinger equation (1.1) with electronic Hamiltonian $h(x, \delta)$ that satisfies hypotheses $\mathbf{H 4}, \mathbf{H 5}, \mathbf{H 7}$. Assume that the solution is characterized asymptotically in the past by

$$
\lim _{t \rightarrow-\infty}\left\|\psi(x, t, \epsilon)-\psi_{j}^{-}(x, t, \epsilon,-)\right\|=0
$$

with

$$
\psi_{j}^{-}(x, t, \epsilon,-)=\phi_{j}(x) \int_{\Delta} \frac{Q(E, \epsilon)}{\sqrt{2 k_{j}(-\infty, E)}} e^{-i t E / \epsilon^{2}} e^{i\left(x k_{j}(-\infty, E)-\omega_{j}^{-}(-\infty, E)\right) / \epsilon^{2}} d E,
$$

where the energy density is supported on the interval $\Delta$, and

$$
Q(E, \epsilon)=e^{-G(E) / \epsilon^{2}} e^{-i J(E) / \epsilon^{2}} P(E, \epsilon)
$$

satisfies $\mathbf{C 1}, \mathbf{C 2}$, and $\mathbf{C 3}$. Let $n=\pi(j)$ be given by (3.27), and let

$$
\begin{aligned}
\alpha(E) & =G(E)+\operatorname{Im}\left(\int_{\zeta} k_{j}(z, E) d z\right), \\
\kappa(E) & =J(E)-\operatorname{Re}\left(\int_{\zeta} k_{j}(z, E) d z\right)+\omega_{n}^{-}(\infty, E) .
\end{aligned}
$$

Assume $E^{*}$ is the unique absolute minimum of $\alpha(\cdot)$ in Int $\Delta$.

Then, there exist $\delta_{0}>0, p>0$ arbitrarily close to 3, and a function $\epsilon_{0}:\left(0, \delta_{0}\right) \rightarrow \mathbb{R}^{+}$, such that for all $\delta<\delta_{0}$ and $\epsilon<\epsilon_{0}(\delta)$, the following asymptotics hold as $t \rightarrow \infty$ :

$$
\begin{aligned}
\psi_{n}^{-}(x, t, \epsilon,+)= & \phi_{n}(x) \frac{e^{-i \theta_{j}(\zeta)} \epsilon^{3 / 2} \pi^{3 / 4}}{\left(\left.\frac{d^{2}}{d k^{2}} \alpha(E(k))\right|_{k^{*}}\right)^{1 / 4}} e^{i S_{+}(t) / \epsilon^{2}} \varphi_{0}\left(A_{+}(t), B_{+}(t), \epsilon^{2}, a_{+}(t), \eta_{+}(t), x\right) \\
& \times P\left(E^{*}, \epsilon\right) \sqrt{k^{*}} e^{-\alpha\left(E^{*}\right) / \epsilon^{2}} e^{-i\left(\kappa\left(E^{*}\right)-k^{* 2} \kappa^{\prime}\left(E^{*}\right)\right) / \epsilon^{2}}+O\left(e^{-\alpha\left(E^{*}\right) / \epsilon^{2}} \epsilon^{p}\right)+O_{\epsilon}(1 / t),
\end{aligned}
$$


where $\varphi_{0}$ is parametrized by

$$
\begin{aligned}
& \eta_{+}=k^{*}=\sqrt{2\left(E^{*}-e_{n}(\infty)\right)}, \quad a_{+}=k^{*} \kappa^{\prime}\left(E^{*}\right), \quad B_{+}=\frac{1}{\sqrt{\left.\frac{d^{2}}{d k^{2}} \alpha(E(k))\right|_{k^{*}}}}, \\
& A_{+}=\left(\left.\frac{d^{2}}{d k^{2}} \alpha(E(k))\right|_{k^{*}}+\left.i \frac{d^{2}}{d k^{2}} \kappa(E(k))\right|_{k^{*}}\right) / \sqrt{\left.\frac{d^{2}}{d k^{2}} \alpha(E(k))\right|_{k^{*}} \quad \text { and }} \\
& S_{+}(t)=t\left(k^{* 2} / 2-e_{n}(\infty)\right) .
\end{aligned}
$$

All error terms are estimated in the $L^{2}(\mathbb{R})$ norm, and the estimate $O\left(e^{-\alpha\left(E^{*}\right) / \epsilon^{2}} \epsilon^{p}\right)$ is uniform in $t$, whereas $O_{\epsilon}(1 / t)$ may depend on $\epsilon$.

\section{Remarks:}

i) All quantities computed from the electronic Hamiltonian $h(x, \delta)$ depend on $\delta$, even though that dependencs is not specified in the notation.

ii) The function $\alpha$ has a unique absolute minimum if $|\Delta|$ and $\delta$ are small enough. See Proposition 5.1. However, in the case of several absolute minima, one simply adds the contributions associated with each of them.

iii) The transitions to states that travel to the left in the future are excluded from our analysis because of the lack of uniformity in $E$ in the semiclassical asymptotics of the relevant elements of the matrix $\mathcal{S}(E, \epsilon)$. At the price of some more technicalities, it should also be possible to accommodate this situation by our methods.

iv) When several avoided-crossings are taken into account and meet the requirements of Theorem [3.1. $c_{n}^{-}(\infty, E, \epsilon)$ with $n=\pi(j)$ is given by a product of exponentials of the same form as those in (5.4). The analysis of this situation is essentially identical to the single avoided crossing situation, mutatis mutandis.

v) Further properties of $\psi_{n}(x, t, \epsilon,+)$ are given below. In particular, the characteristics of the average momentum $k^{*}$ and its behavior as a function of $\delta$ are detailed in Section 5.2. The energy densities corresponding to specific incoming states are studied in Section 6 .

vi) The asymptotics of the incoming wave with the electrons in the state $\phi_{j}$ in the remote past are described by the same integral, with the replacements

$$
\left\{\begin{array}{l}
\gamma_{j} \mapsto 0, \\
\omega_{n}^{-}(\infty, \cdot) \mapsto \omega_{j}^{-}(-\infty, \cdot) \\
\sqrt{2\left(E-e_{n}(\infty)\right)} \mapsto \sqrt{2\left(E-e_{j}(-\infty)\right)} \\
\theta_{j} \mapsto 0
\end{array}\right.
$$

\section{Proof of Theorem 5.1;}

Apart from the $E$-independent factor given by

$$
\frac{\phi_{n}(x)}{\sqrt{2}} e^{-i \theta_{j}(\zeta)},
$$


the asymptotics of (4.5) are determined by the integral

$$
\begin{aligned}
T(\epsilon, x, t)= & \int_{\Delta} \\
& \quad \frac{\tilde{P}(E, \epsilon)}{\left(2\left(E-e_{n}(\infty)\right)\right)^{1 / 4}} \\
& \times e^{-G(E) / \epsilon^{2}} e^{-i(t E+J(E)) / \epsilon^{2}} e^{i \gamma_{j}(E) / \epsilon^{2}} e^{i\left(x \sqrt{2\left(E-e_{n}(\infty)\right)}-\omega_{n}^{-}(\infty, E)\right) / \epsilon^{2}} d E,
\end{aligned}
$$

where $\tilde{P}(E, \epsilon)=P(E, \epsilon)\left(1+O_{E}\left(\epsilon^{2}\right)\right)$ satisfies (5.2),

$$
\begin{aligned}
\gamma_{j}(E) & =\int_{\zeta} k_{j}(z, E) d z, \quad \text { and } \\
\omega_{n}^{-}(\infty, E) & =-\int_{0}^{\infty}\left(\sqrt{2\left(E-e_{n}(y)\right)}-\sqrt{2\left(E-e_{n}(\infty)\right)}\right) d y .
\end{aligned}
$$

The $\left(1+O_{E}\left(\epsilon^{2}\right)\right)$ factor in $\tilde{P}(E, \epsilon)$ comes from Theorem 3.1. Recall that $\gamma_{j}$ and $\omega_{j}^{+}(\infty, \cdot)$ are analytic in a complex neighborhood of $\Delta$, and that $\operatorname{Im} \gamma_{j}(E)$ is a positive, decreasing, convex function of $E$, for $\delta$ sufficiently small.

In terms of the $C^{3}$ functions (5.6) and (5.7) we can write $T(\epsilon, x, t)$ as

$$
T(\epsilon, x, t)=\int_{\Delta} \frac{\tilde{P}(E, \epsilon)}{\left(2(E-e(\infty))^{1 / 4}\right.} e^{-\alpha(E) / \epsilon^{2}} e^{-i(t E+\kappa(E)) / \epsilon^{2}} e^{i\left(x \sqrt{2(E-e(\infty))} / \epsilon^{2}\right.} d E,
$$

where we have dropped the index in the asymptotic eigenvalue $e(\infty)=e_{n}(\infty)$. In Section 7 we analyze the small $\epsilon$ asymptotics of $T$ essentially by Laplace's method. The result is

Lemma 5.1 Let $k(E)=\sqrt{2(E-e(\infty))}$, or equivalently, $E(k)=\frac{k^{2}}{2}+e(\infty)$, and assume that $\alpha(\cdot)$ has a unique absolute minimum $E^{*}$. For sufficiently small $\delta$, this minimum is non-degenerate and satisfies $E^{*} \in$ Int $\Delta$. With $k^{*}=k\left(E^{*}\right)$, there exists $p>0$ arbitrarily close to 3, such that as $\epsilon \rightarrow 0$,

$$
\begin{aligned}
T(\epsilon, x, t)= & \frac{\left.k^{*} \frac{d^{2}}{d k^{2}} \alpha(E(k))\right|_{k^{*}}+i\left(x+k^{* 3} \kappa^{\prime \prime}\left(E^{*}\right)\right)}{\left(\left.\frac{d^{2}}{d k^{2}} \alpha(E(k))\right|_{k^{*}}+i\left(t+\left.\frac{d^{2}}{d k^{2}} \kappa(E(k))\right|_{k^{*}}\right)\right)^{3 / 2}} \\
& \times \epsilon \sqrt{2 \pi} \frac{P\left(E^{*}, \epsilon\right)}{\sqrt{k^{*}}} e^{-\alpha\left(E^{*}\right) / \epsilon^{2}} \exp \left\{-i \frac{\left(t E^{*}+\kappa\left(E^{*}\right)-x k^{*}\right)}{\epsilon^{2}}\right\} \\
& \times \exp \left\{-\frac{\left(x-k^{*}\left(t+\kappa^{\prime}\left(E^{*}\right)\right)\right)^{2}}{2 \epsilon^{2}\left(\left.\frac{d^{2}}{d k^{2}} \alpha(E(k))\right|_{k^{*}}+i\left(t+\left.\frac{d^{2}}{d k^{2}} \kappa(E(k))\right|_{k^{*}}\right)\right.}\right\}+O\left(e^{-\alpha\left(E^{*}\right) / \epsilon^{2}} \epsilon^{p}\right),
\end{aligned}
$$

where the error estimate is in the $L^{2}(\mathbb{R})$ norm, uniformly in $t$.

\section{Remarks:}

i) If there are several absolute minima, one simply adds their contributions to get the asymptotics of $T$.

ii) If $T$ is associated with the incoming wave as $t \rightarrow-\infty$, the formula holds with $E_{0}$ in 
place of $E^{*}, k_{0}=\sqrt{2\left(E_{0}-e(-\infty)\right)}$ in place of $k^{*}$, and the changes (5.9).

iii) If $P$ satisfies $\mathbf{C} 3$ and $P\left(E^{*}, \epsilon\right)=O\left(\epsilon^{d}\right)$ for some $d \geq \frac{3}{2}$, then the above analysis yields no information.

To relate the integral $T$ to standard Born-Oppenheimer states involving normalized free Gaussian states, we must identify (5.10) with (5.5), making use of (5.8), and taking care of the $x$ and $t$ dependence in the non-Gaussian part of (5.10). That is the content of the next lemma which completes the proof of Theorem 5.1 .

With the identifications (5.8), we have

Lemma 5.2 For small $\epsilon$ and $0<p<3$, we have

$$
\begin{aligned}
T(\epsilon, x, t)= & \epsilon^{3 / 2} 2^{1 / 2} \pi^{3 / 4}\left(\left.\frac{d^{2}}{d k^{2}} \alpha(E(k))\right|_{k^{*}}\right)^{-1 / 4} \frac{P\left(E^{*}, \epsilon\right)}{\sqrt{k^{*}}} e^{-\alpha\left(E^{*}\right) / \epsilon^{2}} \\
& \times e^{-i\left(\kappa\left(E^{*}\right)-k^{* 2} \kappa^{\prime}\left(E^{*}\right)\right) / \epsilon^{2}}\left(\frac{\left.k^{*} \frac{d^{2}}{d k^{2}} \alpha(E(k))\right|_{k^{*}}+i\left(x+k^{* 3} \kappa^{\prime \prime}\left(E^{*}\right)\right)}{\left.\frac{d^{2}}{d k^{2}} \alpha(E(k))\right|_{k^{*}}+i\left(t+\left.\frac{d^{2}}{d k^{2}} \kappa(E(k))\right|_{k^{*}}\right)}\right) \\
& \times e^{i S_{+}(t) / \epsilon^{2}} \varphi_{0}\left(A_{+}(t), B_{+}(t), \epsilon^{2}, a_{+}(t), \eta_{+}(t), x\right)+O\left(e^{-\alpha\left(E^{*}\right) / \epsilon^{2}} \epsilon^{p}\right),
\end{aligned}
$$

where the error is estimated in the $L^{2}(\mathbb{R})$ norm, uniformly in $t$.

Furthermore, in the $L^{2}$ norm, for small $\epsilon$ and large $|t|$, we have

$$
\begin{aligned}
T(\epsilon, x, t)= & \epsilon^{3 / 2} 2^{1 / 2} \pi^{3 / 4} P\left(E^{*}, \epsilon\right) \sqrt{k^{*}} e^{-\alpha\left(E^{*}\right) / \epsilon^{2}} e^{-i\left(\kappa\left(E^{*}\right)-k^{* 2} \kappa^{\prime}\left(E^{*}\right)\right) / \epsilon^{2}} \\
& \times \quad e^{i S_{+}(t) / \epsilon^{2}} \varphi_{0}\left(A_{+}(t), B_{+}(t), \epsilon^{2}, a_{+}(t), \eta_{+}(t), x\right)\left(\left.\frac{d^{2}}{d k^{2}} \alpha(E(k))\right|_{k^{*}}\right)^{-1 / 4} \\
& +O\left(e^{-\alpha\left(E^{*}\right) / \epsilon^{2}} \epsilon^{p}\right)+O\left(\epsilon^{3 / 2} /|t|\right),
\end{aligned}
$$

where the first error term is uniform in $t$.

\section{Remarks:}

i) We note that the quantities $\alpha(\cdot), k^{*}$, and $B_{+}$depend only on the index $j$, while $\kappa(\cdot)$, and hence, $A_{+}$depend on both $j$ and $n$.

ii) More detailed computations are carried out in the next section, which is devoted to specific incoming states.

\subsection{Energy and Momentum Shifts}

When there is a single avoided crossing, we can be more precise about the energy and momentum shifts revealed by our general analysis.

For the rest of this section, we assume $h(x, \delta)$ satisfies Hypothesis H6. 
Under this hypothesis, it is known [14] that the decay rate in the Landau-Zener formula (3.29) has the form

$$
\Gamma_{0}(\delta)=\delta^{2} \frac{\pi}{4}\left(\frac{b^{2}}{a}-\frac{c^{2}}{a^{3}}\right)+O\left(\delta^{3}\right) \equiv \delta^{2} D+O\left(\delta^{3}\right),
$$

and that

$$
\operatorname{Im} \theta_{j}\left(\zeta_{j}, \delta\right)=0(\delta)
$$

We use these formulas to get more information on $E^{*}$, the typical energy of the outgoing wave packet, that is determined by the relation

$$
\alpha^{\prime}\left(E^{*}\right)=G^{\prime}\left(E^{*}\right)+\operatorname{Im} \gamma_{j}^{\prime}\left(E^{*}\right)=0
$$

where the primes denote derivatives with respect to $E$.

In the next proposition, we consider two cases:

In the first case, we choose the exponent $G(E)$ in the energy density to be independent of $\delta$. This yields less interesting momentum and energy shifts since they vanish to leading order in $\delta$ as $\delta \rightarrow 0$, in keeping with [10].

In the second case, we choose $G(E)$ to depend on $\delta$ in such a way that the incoming wave packet contains a sufficiently wide spectrum of energies as $\delta \rightarrow 0$. This implies non-trivial

behavior of the relevant quantities to leading order in $\delta$. For obvious reasons, we restore $\delta$ in the notation of this discussion.

Proposition 5.1 Let

$$
\begin{aligned}
& G(E)=g\left(E-E_{0}\right)^{2} / 2+O\left(E-E_{0}\right)^{3} \\
& \operatorname{Im} \gamma_{j}(E, \delta)=\frac{\Gamma_{0}(\delta)}{k_{c}(E)}+O\left(\delta^{3}\right)=\frac{D \delta^{2}}{k_{c}(E)}+O\left(\delta^{3}\right), \quad \text { and } \\
& \alpha(E, \delta)=G(E)+\operatorname{Im} \gamma_{j}(E, \delta),
\end{aligned}
$$

as above.

i) Assume $G$ is independent of $\delta$. Then, for $E^{*}$ defined by (5.11), we have

$$
E^{*}(\delta)=E_{0}+\frac{\Gamma_{0}(\delta)}{g k_{c}^{3}\left(E_{0}\right)}+O\left(\delta^{3}\right)
$$

as $\delta \rightarrow 0$. In this case,

$$
\begin{aligned}
& \alpha\left(E^{*}(\delta)\right)=\frac{\Gamma_{0}(\delta)}{k_{c}\left(E_{0}\right)}+O\left(\delta^{3}\right)>\alpha\left(E_{0}\right), \quad \text { and } \\
& \alpha^{\prime \prime}\left(E^{*}(\delta)\right)= g+O\left(\delta^{2}\right) . \\
& \text { If } G(E)=g\left(E-E_{0}\right)^{2} / 2+g_{1}\left(E-E_{0}\right)^{3} / 6+O\left(E-E_{0}\right)^{4}, \text { then } \\
& \alpha^{\prime \prime}\left(E^{*}(\delta)\right)=g+g_{1} \frac{\Gamma_{0}(\delta)}{g k_{c}^{3}\left(E_{0}\right)}+3 \frac{\Gamma_{0}(\delta)}{k_{c}^{5}\left(E_{0}\right)}+O\left(\delta^{3}\right) .
\end{aligned}
$$


ii) Assume $G(E, \delta)=L\left(\delta\left(E-E_{0}\right)\right)$, for some function $L$, such that

$$
G(E, \delta)=g_{0} \delta^{2}\left(E-E_{0}\right)^{2} / 2+O\left(\delta^{3}\right),
$$

for some $g_{0}>0$, uniformly for $E \in \Delta$. Then

$$
E^{*}(\delta)=E_{1}+O(\delta),
$$

where $0<E_{1}=E_{1}\left(D / g_{0}\right)$ is the unique solution to the equation

$$
\left(E_{1}-E_{0}\right)=D /\left(g_{0} k_{c}^{3}\left(E_{1}\right)\right),
$$

and is independent of $\delta$. In this case,

$$
\begin{aligned}
\alpha\left(E^{*}(\delta)\right) & =\delta^{2}\left(\frac{D}{k_{c}\left(E_{1}\right)}+g_{0}\left(E_{1}-E_{0}\right)^{2} / 2\right)+O\left(\delta^{3}\right) \\
& =\delta^{2}\left(D^{2 / 3} g_{0}^{1 / 3}\left(E_{1}-E_{0}\right)^{1 / 3}+g_{0}\left(E_{1}-E_{0}\right)^{2} / 2\right)+O\left(\delta^{3}\right) \\
& >\alpha\left(E_{0}\right), \quad \text { and } \\
\alpha^{\prime \prime}\left(E^{*}(\delta)\right) & =\delta^{2} g_{0}+3 \frac{\Gamma_{0}(\delta)}{k_{c}^{5}\left(E_{0}\right)}+O\left(\delta^{3}\right) .
\end{aligned}
$$

Proof: Both statements follow from application of the Implicit Function Theorem and the observation that $\alpha$ is a strictly convex function of $E$ on $\Delta$.

\section{Remarks:}

a) The first result shows no effect to leading order in $\delta$ in the exponential decay rate of transition probability. The value of $E^{*}(\delta)$ and the width of the outgoing wave packet can be computed. Their variations with respect to the corresponding quantities in the incoming wave packet are of order $\delta^{2}$, and hence, are rather small.

b) In case ii) of the proposition, the equation that determines $E^{*}$ can be rewritten as the quintic equation

$$
k_{c}^{5}(E)-k_{c}^{3}(E) k_{c}^{2}\left(E_{0}\right)-2 D / g_{0}=0 .
$$

c) In the case ii), the variation of exponential decay rate in the transition probability is given by

$$
\begin{aligned}
& \alpha\left(E^{*}(\delta)\right)-\alpha\left(E_{0}\right)=\delta^{2}\left(D\left(\frac{1}{k_{c}\left(E_{1}\right)}-\frac{1}{k_{c}\left(E_{0}\right)}\right)+g_{0}\left(E_{1}-E_{0}\right)^{2} / 2\right)+O\left(\delta^{3}\right) \\
& =\frac{\delta^{2} D}{2 g_{0} k_{c}\left(E_{1}\right)^{6} k_{c}\left(E_{0}\right)}\left(2 g_{0} k_{c}\left(E_{1}\right)^{5}\left(k_{c}\left(E_{0}\right)-k_{c}\left(E_{1}\right)\right)+D k_{c}\left(E_{0}\right)\right)+O\left(\delta^{3}\right) .
\end{aligned}
$$

d) The results above hold provided one knows $E^{*}$ is the unique absolute minimum of $G$ in the set $\Delta$, which is generically true. Again, if there are several minima, one simply adds the corresponding contributions. 


\section{$6 \quad$ Energy Densities and Transitions when the Incoming State has the form $\varphi_{m}$}

In this section we study the special case in which the incoming state is asymptotically in the past on electronic level $j$ with the nuclear wave function given by one of the functions $\varphi_{m}$. For simplicity, we restrict attention to wave packets that are incoming from the left.

In the simplest situation, the incoming wave packet is asmyptotic to

$$
e^{i\left(\eta_{-}^{2} / 2-e_{j}(-\infty)\right) t / \epsilon} \varphi_{0}\left(A_{-}+i t B_{-}, B_{-}, \epsilon^{2}, a_{-}+\eta_{-} t, \eta_{-}, x\right) \phi_{j}(x),
$$

as $t \rightarrow-\infty$. Here we choose $\eta_{-}>0$ and the set $\Delta$, so that $\eta_{-}^{2} / 2+e_{j}(-\infty)$ is in the interior of $\Delta$, and that the minimum of $\Delta$ lies strictly above the spectrum of $h(x)$ for all $x$.

We choose a smooth cut-off function $F(E)$ whose support is a subset of the interior of $\Delta$, which takes the value 1 on an interval whose interior contains $\eta_{-}^{2} / 2+e_{j}(-\infty)$, and whose length is almost as large as that of $\Delta$.

From our assumptions on $\Delta$, there is a one-to-one correspondence between $E \in \Delta$ and positive $k$, such that $k^{2} / 2+e_{j}(-\infty)=E$. For $E \in \Delta$, we make the change of variables from $k$ to $E$ at $t=0$ in the (rescaled) Fourier transform of the Gaussian in (6.1) (see [9]). Taking into account the normalization (3.7) of the generalized eigenvectors, this leads to the energy density

$$
\begin{aligned}
Q(E, \epsilon) & =\frac{F(E) e^{i \omega_{j}^{-}(E,-\infty) / \epsilon^{2}}}{\epsilon \sqrt{\pi k(E)}} \varphi_{0}\left(B_{-}, A_{-}, \epsilon^{2}, \eta_{-},-a_{-}, k(E)\right) \\
& =\frac{F(E) e^{i \omega_{j}^{-}(E,-\infty) / \epsilon^{2}}}{\epsilon \sqrt{\pi \sqrt{2\left(E-e_{j}(-\infty)\right)}}} \varphi_{0}\left(B_{-}, A_{-}, \epsilon^{2}, \eta_{-},-a_{-}, \sqrt{2\left(E-e_{j}(-\infty)\right)}\right)
\end{aligned}
$$

that we use in (4.2).

Since $\eta_{-}^{2} / 2+e_{j}(-\infty)$ is in the interior of the set where $F(E)=1$, the wave functions (6.1) and $\psi(x, t, \epsilon,-)$ defined by (4.2) with the energy density defined by (6.2) differ in $L^{2}(\mathbb{R})$ norm by an $O\left(e^{-C / \epsilon^{2}}\right)$ error. To be sure that this error is smaller than the non-adiabatic effect we are studying, we assume any one of the following conditions:

1. Take the avoided crossing gap $\delta$ to be small enough that the non-adiabatic effect is larger than the error we make here.

2. Choose $\left|B_{-}\right|$to be sufficiently small. That increases the value of $C$ in this error estimate.

3. Fix the minimum of $\Delta$, but then choose $\eta_{-}$large enough so that the cut off is farther out in the tail of the Gaussian in momentum space. This also makes the non-adiabatic effect larger since $\eta_{-}$is larger.

With $Q(E, \epsilon)$ chosen by (6.2), we have in the notation of (5.1),

$$
\begin{aligned}
G(E) & =\left(\operatorname{Re}\left(A_{-} / B_{-}\right)\right) \frac{\left(\sqrt{2\left(E-e_{j}(-\infty)\right)}-\eta_{-}\right)^{2}}{2} \\
& =\left|B_{-}\right|^{-2} \frac{\left(\sqrt{2\left(E-e_{j}(-\infty)\right)}-\eta_{-}\right)^{2}}{2},
\end{aligned}
$$




$$
\begin{gathered}
J(E)=\left(\operatorname{Im}\left(A_{-} / B_{-}\right)\right) \frac{\left(\sqrt{2\left(E-e_{j}(-\infty)\right)}-\eta_{-}\right)^{2}}{2} \\
+a_{-}\left(\sqrt{2\left(E-e_{j}(-\infty)\right)}-\eta_{-}\right)-\omega_{j}^{-}(E,-\infty), \\
P(E, \epsilon)=\pi^{-3 / 4} \epsilon^{-3 / 2} B_{-}^{-1 / 2}\left(2\left(E-e_{j}(-\infty)\right)\right)^{-1 / 4} F(E) .
\end{gathered}
$$

Also, conditions $\mathbf{C 1}$, and $\mathbf{C 2}$ are satisfied, and provided we remove the trivial normalization factor of $\epsilon^{-3 / 2}$ from $P(E, \epsilon)$, then condition C3 is also satisfied.

We already know that asymptotically in the past, the interacting wave function determined by (6.2) agrees with (6.1) up to an $O\left(e^{-C / \epsilon^{2}}\right)$ error, and we observe that the density $Q(E, \epsilon)$ is sharply peaked around the energy

$$
E_{0}=\frac{\eta_{-}^{2}}{2}+e_{j}(-\infty) \quad \text { corresponding to } \quad \eta_{-}=\sqrt{2\left(E_{0}-e_{j}(-\infty)\right)}
$$

Thus from (6.3), we see that

$$
G(E)=\frac{\left(E-E_{0}\right)^{2}}{2\left(\eta_{-}\left|B_{-}\right|\right)^{2}}+O\left(\left(E-E_{0}\right)^{3}\right), \quad \text { i.e., } \quad g=\frac{1}{\left(\eta_{-}\left|B_{-}\right|\right)^{2}}
$$

We are not particularly interested in the main component of the wave function for large time that has not made a non-adiabatic transition. However, by a similar analysis, it could be determined by our techniques. Of course, it is what one would expect from the standard time-dependent Born-Oppenheimer approximation.

Our focus is on the dominant non-adiabatic component, which is determined to leading order in $\epsilon$ by Theorem 5.1. From the above calculations and Theorem 5.1, we immediately get our main result for Gaussian incoming states:

Theorem 6.1 Assume Hypotheses $\mathbf{H 4}, \mathbf{H 5}$, and $\mathbf{H 7}$, and assume $\Delta, A_{-}, B_{-}, a_{-}, \eta_{-}, \delta$, and the levels $j$ and $n$ have been chosen to satisfy the requirements above. Let $\Psi(x, \epsilon, t)$ be the solution to the Schrödinger equation that is asymptotic as $t \rightarrow-\infty$ to

$$
e^{i\left(\eta_{-}^{2} / 2-e_{j}(-\infty)\right) t / \epsilon^{2}} \varphi_{0}\left(A_{-}+i t B_{-}, B_{-}, \epsilon^{2}, a_{-}+\eta_{-} t, \eta_{-}, x\right) \phi_{j}(x) .
$$

The leading non-adiabatic component of $\Psi(x, \epsilon, t)$ as $t \rightarrow \infty$ and $\epsilon \rightarrow 0$ in $L^{2}$ norm is on electronic level $\phi_{n}(x)$ and is given by

$$
\mathcal{A}_{n j}(\epsilon) e^{i\left(\eta_{+}^{2} / 2-e_{n}(\infty)\right) t / \epsilon^{2}} \varphi_{0}\left(A_{+}+i t B_{+}, B_{+}, \epsilon^{2}, a_{+}+\eta_{+} t, \eta_{+}, x\right) \phi_{n}(x),
$$

where the values of $A_{+}, B_{+}, a_{+}, \eta_{+}=k^{*}$ are those given by (5.8) as in Theorem 5.1. The amplitude for making this transition from level $j$ to level $n$ is given by

$$
\mathcal{A}_{n j}(\epsilon)=e^{-i \theta_{j}(\zeta)} e^{-\alpha\left(E^{*}\right) / \epsilon^{2}} \sqrt{\frac{B_{+}}{B_{-}}} e^{-i\left(\kappa\left(E^{*}\right)-k^{*} a_{+}\right) / \epsilon^{2}} .
$$


In particular

$$
\begin{aligned}
B_{+} & =\left(\left(G^{\prime \prime}\left(E^{*}\right)+\operatorname{Im} \gamma_{j}^{\prime \prime}\left(E^{*}\right)\right) k^{* 2}\right)^{-1 / 2}=\left(\frac{\eta_{-}}{\left|B_{-}\right|^{2} k^{*}}+\operatorname{Im} \gamma_{j}^{\prime \prime}\left(E^{*}\right) k^{* 2}\right)^{-1 / 2} \\
& =\frac{\left|B_{-}\right|}{\sqrt{\frac{\eta_{-}}{k^{*}}+\operatorname{Im} \gamma_{j}^{\prime \prime}\left(E^{*}\right)\left|B_{-}\right| k^{* 2}}}
\end{aligned}
$$

Remark: Depending on the relative size of $\left|B_{-}\right|$with respect to $\delta$, we can apply Proposition 5.1 to further characterize $\mathcal{A}_{n j}$.

We now turn our attention to the situation where the incoming nuclear wave packet is in the state $\varphi_{m}$. The only change from the situation just considered is that we must replace the function $P(E, \epsilon)$ in (6.5) by

$$
\begin{aligned}
P(E, \epsilon)= & (-i)^{m} 2^{-m / 2}(m !)^{-1 / 2} \pi^{-3 / 4} \epsilon^{-3 / 2}\left(2\left(E-e_{j}(-\infty)\right)\right)^{-1 / 4} B_{-}^{-(m+1) / 2}\left(\overline{B_{-}}\right)^{m / 2} \\
& \times \quad H_{m}\left(\frac{\sqrt{2\left(E-e_{j}(-\infty)\right)}-\eta_{-}}{\epsilon|B|}\right) F(E) .
\end{aligned}
$$

Again, this satisfies Condition $\mathbf{C} 3$ if we take out the trivial factor of $\epsilon^{-(m+3 / 2)}$.

Theorem 6.2 Assume the Hypotheses of Theorem [6.1. Let $\Psi(x, \epsilon, t)$ be the solution to the Schrödinger equation that is asymptotic as $t \rightarrow-\infty$ to

$$
e^{i\left(\eta_{-}^{2} / 2-e_{j}(-\infty)\right) t / \epsilon^{2}} \varphi_{m}\left(A_{-}+i t B_{-}, B_{-}, \epsilon^{2}, a_{-}+\eta_{-} t, \eta_{-}, x\right) \phi_{j}(x) .
$$

The leading non-adiabatic component of $\Psi(x, \epsilon, t)$ as $t \rightarrow \infty$ and $\epsilon \rightarrow 0$ in $L^{2}$ norm is on electronic level $\phi_{n}(x)$, and is given by

$$
\mathcal{A}_{n j}^{(m)}(\epsilon) e^{i\left(\eta_{+}^{2} / 2-e_{n}(\infty)\right) t / \epsilon^{2}} \varphi_{0}\left(A_{+}+i t B_{+}, B_{+}, \epsilon^{2}, a_{+}+\eta_{+} t, \eta_{+}, x\right) \phi_{n}(x),
$$

where the values of $A_{+}, B_{+}, a_{+}, \eta_{+}=k^{*}$ are those given by (5.8), as in Theorem [5.1. The amplitude for making the transition from level $j$ to level $n$ is given by

$$
\begin{aligned}
\mathcal{A}_{n j}^{(m)}(\epsilon) & =e^{-i \theta_{j}(\zeta)} e^{-\alpha\left(E^{*}\right) / \epsilon^{2}} \sqrt{\frac{B_{+}}{B_{-}}} \frac{e^{-i\left(\kappa\left(E^{*}\right)-k^{*} a_{+}\right) / \epsilon^{2}}}{2^{m / 2}(m !)^{1 / 2}}\left(\frac{\overline{B_{-}}}{B_{-}}\right)^{m / 2} H_{m}\left(\frac{k^{*}-\eta_{-}}{\epsilon\left|B_{-}\right|}\right) \\
& =e^{-i \theta_{j}(\zeta)} \frac{e^{-\alpha\left(E^{*}\right) / \epsilon^{2}}}{\epsilon^{m}} \sqrt{\frac{B_{+}}{B_{-}}} \frac{e^{-i\left(\kappa\left(E^{*}\right)-k^{*} a_{+}\right) / \epsilon^{2}}}{(m !)^{1 / 2}}\left(\frac{\sqrt{2}\left(k^{*}-\eta_{-}\right)}{B_{-}}\right)^{m}(1+O(\epsilon)) .
\end{aligned}
$$

In particular, $B_{+}$is again given by (6.6) and the pre-exponential factor is of order $\epsilon^{-m}$. 


\section{Technicalities}

Proof of lemma 13.1; We consider only the limit $x \rightarrow \infty$ and the choice $\sigma=+$. The other cases are similar. In this proof, $c_{n}$ denotes a finite constant that depends only on $n$, but may vary from line to line.

Explicitly, for any $n \in \mathbf{N}$,

$$
\frac{\partial^{n}}{\partial E^{n}} \sqrt{2\left(E-e_{j}(x)\right)}=c_{n}\left(2\left(E-e_{j}(x)\right)\right)^{1 / 2-n}
$$

uniformly for $E \in \Delta$. So, the first assertion is true. Moreover,

$$
\begin{aligned}
& \frac{\partial^{n}}{\partial E^{n}}\left(\sqrt{2\left(E-e_{j}(x)\right)}-\sqrt{2\left(E-e_{j}(\infty)\right)}\right)= \\
& c_{n}\left(\left(2\left(E-e_{j}(x)\right)\right)^{1 / 2-n}-\left(2\left(E-e_{j}(\infty)\right)\right)^{1 / 2-n}\right) .
\end{aligned}
$$

For $n=0$, we have by (2.2),

$$
\begin{aligned}
\sqrt{2\left(E-e_{j}(x)\right)}-\sqrt{2\left(E-e_{j}(\infty)\right)} & =\frac{2\left(e_{j}(\infty)-e_{j}(x)\right)}{\sqrt{2\left(E-e_{j}(x)\right)}+\sqrt{2\left(E-e_{j}(\infty)\right)}} \\
& =O\left(e_{j}(\infty)-e_{j}(x)\right)=O\left(<x>^{-(2+\nu)}\right) .
\end{aligned}
$$

For $n>0$, we can write

$$
\begin{aligned}
& \left(2\left(E-e_{j}(x)\right)\right)^{1 / 2-n}-\left(2\left(E-e_{j}(\infty)\right)\right)^{1 / 2-n} \\
= & \frac{\left(2\left(E-e_{j}(x)\right)\right)^{1 / 2}-\left(2\left(E-e_{j}(\infty)\right)\right)^{1 / 2}}{\left(2\left(E-e_{j}(x)\right)\right)^{n}} \\
& +\left(\sum_{k=0}^{n-1} \frac{\left(2\left(E-e_{j}(\infty)\right)\right)^{1 / 2}}{\left(2\left(E-e_{j}(x)\right)\right)^{k+1}\left(2\left(E-e_{j}(\infty)\right)\right)^{n-k}}\right)\left(2\left(e_{j}(x)-e_{j}(\infty)\right),\right.
\end{aligned}
$$

to which the estimate (17.1) applies. The second assertion follows.

By definition,

$$
r_{j}^{+}(+, x, \epsilon)=-\int_{x}^{\infty}\left(\left(2\left(E-e_{j}(y)\right)\right)^{1 / 2}-\left(2\left(E-e_{j}(\infty)\right)\right)^{1 / 2}\right) d y,
$$

so that (7.2) implies the estimates on $\frac{\partial^{n}}{\partial E^{n}} r_{j}^{+}(+, x, E)$.

We now study the properties of the $c_{j}^{\tau}$ 's. Again, we shall consider $x \rightarrow+\infty$; the other case is similar. We first compute

$$
\begin{aligned}
a_{j l}^{\tau \sigma}(x, E)= & -\frac{1}{2} \frac{1}{\sqrt{k_{j}(x, E) k_{l}(x, E)}}\left(\left\langle\phi_{j}(x), \phi_{l}^{\prime}(x)\right\rangle\left(k_{j}(x, E)+\tau \sigma k_{l}(x, E)\right)\right. \\
& \left.+\left(\sigma \tau-\frac{k_{j}}{k_{l}}\right) \frac{\left\langle\phi_{j}(x), \phi_{l}(x)\right\rangle}{2} \frac{\partial}{\partial x} k_{l}(x, E)\right) \\
=- & -\frac{1}{2} \frac{1}{\sqrt{k_{j}(x, E) k_{l}(x, E)}}\left(\left\langle\phi_{j}(x), \phi_{l}^{\prime}(x)\right\rangle\left(k_{j}(x, E)+\tau \sigma k_{l}(x, E)\right)\right. \\
& \left.+\left(\sigma \tau-\frac{k_{j}}{k_{l}}\right) \frac{\left\langle\phi_{j}(x), \phi_{l}(x)\right\rangle}{2 k_{l}(x, E)} e_{l}^{\prime}(x)\right) .
\end{aligned}
$$


The presence of the factors $\left\langle\phi_{j}(x), \phi_{l}^{\prime}(x)\right\rangle$ and $e_{l}^{\prime}(x)$, which are independent of $E$ and decay as $1 /\langle x\rangle^{2+\nu}$, implies together with (3.17) that

$$
\frac{\partial^{n}}{\partial E^{n}} a_{j l}^{\tau \sigma}(x, E)=O\left(<x>^{-(2+\nu)}\right) .
$$

We denote the coefficients $c_{j}^{\tau}$ collectively by

$$
\mathbf{c}(x, E, \epsilon)=\left(\begin{array}{l}
\mathbf{c}^{+}(x, E, \epsilon) \\
\mathbf{c}^{-}(x, E, \epsilon)
\end{array}\right) \in \mathbb{C}^{2 m}
$$

and the generator of equation (3.12) by the $2 m \times 2 m$ block matrix

$$
\begin{aligned}
& \mathbf{M}(x, E, \epsilon)=
\end{aligned}
$$

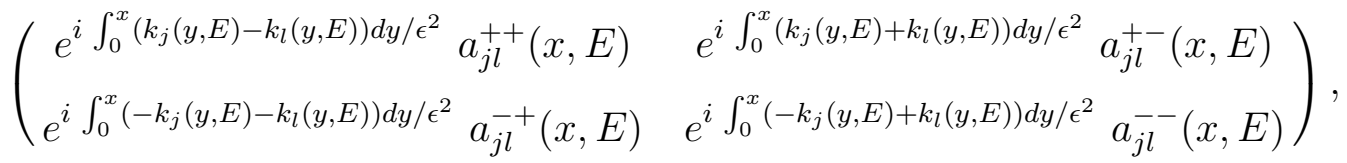

so that (3.12) can be rewritten as

$$
\frac{\partial}{\partial x} \mathbf{c}(x, E, \epsilon)=\mathbf{M}(x, E, \epsilon) \mathbf{c}(x, E, \epsilon)
$$

Expressing the solutions as Dyson series, we obtain

$$
\begin{aligned}
\mathbf{c}(x, E, \epsilon)= & \sum_{n=0}^{\infty} \int_{0}^{x} \int_{0}^{x_{1}} \cdots \int_{0}^{x_{n-1}} \\
& \times \mathbf{M}\left(x_{1}, E, \epsilon\right) \mathbf{M}\left(x_{2}, E, \epsilon\right) \cdots \mathbf{M}\left(x_{n}, E, \epsilon\right) d x_{1} d x_{2} \cdots d x_{n} \mathbf{c}(0, E, \epsilon)
\end{aligned}
$$

where, because of (7.4), $\int_{0}^{\infty}\|\mathbf{M}(y, E, \epsilon)\| d y<\infty$, uniformly for $E \in \Delta$, we get the usual bound

$$
\|\mathbf{c}(x, E, \epsilon)\| \leq e^{\int_{0}^{\infty}\|\mathbf{M}(y, E, \epsilon)\| d y}\|\mathbf{c}(0, E, \epsilon)\| .
$$

By showing that $\|c(x, E, \epsilon)-c(y, E, \epsilon)\|$ is arbitrarily small for large $x$ and $y$, we see that $\lim _{x \rightarrow \infty} \mathbf{c}(x, E, \epsilon)=\mathbf{c}(\infty, E, \epsilon)$ exists. Because of the presence of the phases $e^{i \int_{0}^{x}\left(\tau k_{j}(y, E)+\sigma k_{l}(y, E)\right) d y / \epsilon^{2}}$ in $\mathbf{M}(x, E, \epsilon)$, whose derivatives with respect to $E$ satisfy

$$
\frac{\partial^{n}}{\partial E^{n}} e^{i \int_{0}^{x}\left(\tau k_{j}(y, E)+\sigma k_{l}(y, E)\right) d y / \epsilon^{2}}=O\left(<x>^{n}\right)
$$

we get for $n=0,1$,

$$
\frac{\partial^{n}}{\partial E^{n}} \mathbf{M}(x, E, \epsilon)=O\left(<x>^{-(2+\nu-n)}\right) .
$$

Thus, by the Lebesgue Dominated Convergence Theorem, we get from (7.5) that, as $x \rightarrow \infty$ and uniformly for $E \in \Delta$,

$$
\frac{\partial^{n}}{\partial E^{n}} \mathbf{c}(x, E, \epsilon)=O(1), \quad \text { for } \quad n=0,1
$$


Finally, we consider

$$
\mathbf{c}(x, E, \epsilon)-\mathbf{c}(\infty, E, \epsilon)=-\int_{x}^{\infty} \mathbf{M}(y, E, \epsilon) \mathbf{c}(y, E, \epsilon) d y
$$

In this expression, we use the above properties of $\mathbf{M}$, $\mathbf{c}$, and their derivatives to obtain the last two statements of the lemma.

\section{Proof of Lemma 4.1;}

We assume $t \neq 0$ and rewrite the exponential factors in (4.1) as

$$
e^{-i\left(\int_{0}^{x} k_{j}^{\sigma}(y, E) d y+t E\right) / \epsilon^{2}}=i \epsilon^{2} \frac{\frac{\partial}{\partial E} e^{-i\left(\int_{0}^{x} k_{j}^{\sigma}(y, E) d y+t E\right) / \epsilon^{2}}}{\left(t+\int_{0}^{x} \frac{\partial}{\partial E} k_{j}^{\sigma}(y, E) d y\right)} .
$$

Then, for each integral in (4.1), we have

$$
\begin{aligned}
& \int_{\Delta} \frac{Q(E, \epsilon) c_{j}^{\sigma}(x, E, \epsilon)}{\sqrt{k_{j}(x, E)}} e^{-i\left(\int_{0}^{x} k_{j}^{\sigma}(y, E) d y+t E\right) / \epsilon^{2}} d E \\
& =\left.i \epsilon^{2} e^{-i\left(\int_{0}^{x} k_{j}^{\sigma}(y, E) d y+t E\right) / \epsilon^{2}} \frac{Q(E, \epsilon) c_{j}^{\sigma}(x, E, \epsilon)}{\sqrt{k_{j}(x, E)}\left(t+\int_{0}^{x} \frac{\partial}{\partial E} k_{j}^{\sigma}(y, E) d y\right)}\right|_{E_{1}} ^{E_{2}} \\
& -\int_{\Delta} \frac{\partial}{\partial E}\left\{\frac{Q(E, \epsilon) c_{j}^{\sigma}(x, E, \epsilon)}{\sqrt{k_{j}(x, E)}\left(t+\int_{0}^{x} \frac{\partial}{\partial E} k_{j}^{\sigma}(y, E) d y\right)}\right\} i \epsilon^{2} e^{-i\left(\int_{0}^{x} k_{j}^{\sigma}(y, E) d y+t E\right) / \epsilon^{2}} d E .
\end{aligned}
$$

The quantities $c_{j}^{\sigma}(x, E, \epsilon), \sqrt{k_{j}(x, E)}$, and their derivatives with respect to $E$ are uniformly bounded in $x$ and $E$. Also,

$$
\begin{aligned}
\int_{0}^{x} \frac{\partial k_{j}^{\sigma}(y, E)}{\partial E} d y & =x \frac{\partial k_{j}^{\sigma}( \pm \infty, E)}{\partial E}+O(1) \\
& =\frac{\sigma x}{k_{j}( \pm \infty, E)}+O(1)
\end{aligned}
$$

uniformly for $E \in \Delta$ as $x \rightarrow \pm \infty$.

Thus, the boundary terms in (7.7) satisfy

$$
\begin{aligned}
& \left.i \epsilon^{2} e^{-i\left(\int_{0}^{x} k_{j}^{\sigma}(y, E) d y+t E\right) \epsilon^{2}} \frac{Q(E, \epsilon) c_{j}^{\sigma}(x, E, \epsilon)}{\sqrt{k_{j}(x, E)}\left(t+\int_{0}^{x} \frac{\partial}{\partial E} k_{j}^{\sigma}(y, E) d y\right)}\right|_{E_{1}} ^{E_{2}} \\
= & O\left(\frac{1}{\left|k_{j}^{\sigma}\left( \pm \infty, E_{1}\right) t+x+O(1)\right|}+\frac{1}{\left|k_{j}^{\sigma}\left( \pm \infty, E_{2}\right) t+x+O(1)\right|}\right) .
\end{aligned}
$$


We now apply the restrictions on $x / t$ in the statement of the Lemma. For any choice of $j$ and $\sigma$, they ensure that the denominators on the right hand side of (7.8) can be estimated, uniformly in $E$ and for large $|x|$, by

$$
\begin{aligned}
\left|k_{j}^{\sigma}( \pm \infty, E) t+x+O(1)\right| & =|x|\left|1+k_{j}^{\sigma}( \pm \infty, E) t / x+O(1 / x)\right| \\
& \geq|x|(\alpha+O(1 /|x|)),
\end{aligned}
$$

where $\alpha$ is the number that appears in the statement of the lemma. From this, we see that the boundary terms in (17.7) are $O(1 /|x|)$.

We estimate the integral term in (7.7) in a similar way. Under the restrictions on $x / t$ in the lemma, we obtain

$$
\begin{gathered}
\int_{\Delta} i \epsilon^{2} e^{-i\left(\int_{0}^{x} k_{j}^{\sigma}(y, E) d y+t E\right) / \epsilon^{2}}\left(\left\{\frac{\frac{\partial}{\partial E}\left(Q(E, \epsilon)\left(k_{j}^{\sigma}(x, E)\right)^{-1 / 2} c_{j}^{\sigma}(x, E, \epsilon)\right)}{t+\int_{0}^{x} \frac{\partial}{\partial E} k_{j}^{\sigma}(y, E) d y}\right\}\right. \\
\left.-\frac{Q(E, \epsilon)\left(k_{j}^{\sigma}(x, E)\right)^{-1 / 2} c_{j}^{\sigma}(x, E, \epsilon) \int_{0}^{x} \frac{\partial^{2}}{\partial E^{2}} k_{j}^{\sigma}(y, E) d y}{\left(t+\int_{0}^{x} \frac{\partial}{\partial E} k_{j}^{\sigma}(y, E) d y\right)^{2}}\right) d E \\
=O(1 /|x|) .
\end{gathered}
$$

This implies the lemma for $t \neq 0$. When $t=0$, the estimate (17.8) with $t=0$ yields the result in a more direct way.

Proof of Proposition 4.1; We can write

$$
\begin{aligned}
& \psi(x, t, \epsilon)-\psi(x, t, \epsilon, \pm)=\sum_{j=1,2 \sigma= \pm} \phi_{j}(x) \times \\
& \times\left\{\int_{\Delta} \frac{Q(E, \epsilon) d E}{\sqrt{2 k_{j}( \pm \infty, E)}} e^{-i t E / \epsilon^{2}}\left(c_{j}^{\sigma}(x, E, \epsilon)-c_{j}^{\sigma}( \pm \infty, E, \epsilon)\right) e^{-i \int_{0}^{x} k_{j}^{\sigma}(y, E) d y / \epsilon^{2}}\right. \\
& \quad+\int_{\Delta} \frac{Q(E, \epsilon) d E}{\sqrt{2 k_{j}( \pm \infty, E)}} e^{-i t E / \epsilon^{2}} c_{j}^{\sigma}( \pm \infty, E, \epsilon) \\
& \left.\quad+\int_{\Delta} \frac{Q(E, \epsilon) c_{j}^{\sigma}(x, E, \epsilon) d E}{\sqrt{k_{j}( \pm \infty, E) k_{j}(x, E)}} e^{-i t E / \epsilon^{2}} \frac{\left(e^{-i \int_{0}^{x} k_{j}^{\sigma}(y, E) d y / \epsilon^{2}}-e^{-i\left(x k_{j}^{\sigma}( \pm \infty, E)+\omega_{j}^{\sigma}( \pm \infty, E)\right) / \epsilon^{2}}\right)}{\sqrt{2 k_{j}( \pm \infty, E)}+\sqrt{2 k_{j}(x, E)}} e^{-i \int_{0}^{x} k_{j}^{\sigma}(y, E) d y / \epsilon^{2}}\right\} .
\end{aligned}
$$

The first step of the proof consists of integrating by parts to get a factor of $1 / t$ according to

$$
\begin{aligned}
\int_{\Delta} f(x, E, \epsilon) e^{-i t E / \epsilon^{2}} d E= & \left.\frac{i \epsilon^{2}}{t} f(x, E, \epsilon) e^{-i t E / \epsilon^{2}}\right|_{E_{1}} ^{E_{2}} \\
& -\frac{i \epsilon^{2}}{t} \int_{\Delta} \frac{\partial}{\partial E} f(x, E, \epsilon) e^{-i t E / \epsilon^{2}} d E .
\end{aligned}
$$


We then bound the $L^{2}\left(\mathbb{R}_{x}\right)$ norm of each term that arises from these integrations by parts, with bounds that are uniform in $t$.

From the estimates in Lemma 3.1. we see that all the boundary terms in (17.10) coming from (7.9) are of order $\langle x\rangle^{-(1+\nu)}$. Thus, their $L^{2}$ norms are bounded, uniformly in $t$. The integral terms in (7.10) coming from (7.9) all have the form

$$
\int_{\Delta} g_{j}(x, E, \epsilon) e^{-i\left(\int_{0}^{x} k_{j}^{\sigma}(y, E) d y+t E\right) / \epsilon^{2}} d E, \quad j=1,2,3,
$$

where the first integral from (17.9) contains the function

$$
\begin{aligned}
g_{1}(x, E, \epsilon) & =\left\{\frac{\partial}{\partial E}\left(\frac{Q(E, \epsilon)}{\sqrt{k_{j}( \pm \infty, E)}}\left(c_{j}^{\sigma}(x, E, \epsilon)-c_{j}^{\sigma}( \pm \infty, E, \epsilon)\right)\right)\right\} \\
& -i \frac{Q(E, \epsilon)}{\sqrt{k_{j}( \pm \infty, E)}}\left(c_{j}^{\sigma}(x, E, \epsilon)-c_{j}^{\sigma}( \pm \infty, E, \epsilon)\right) \int_{0}^{x} \frac{\partial}{\partial E} k_{j}^{\sigma}(y, E) d y .
\end{aligned}
$$

With the notation of Lemma 3.1 the second integral in (17.9) contains the function

$$
\begin{aligned}
g_{2}(x, E, \epsilon) & =\frac{\partial}{\partial E}\left(\frac{Q(E, \epsilon)}{\sqrt{k_{j}( \pm \infty, E)}} c_{j}^{\sigma}( \pm \infty, E, \epsilon)\left(1-e^{i\left(r_{j}^{\sigma}( \pm, x, E)\right) / \epsilon^{2}}\right)\right) \\
& -i \frac{Q(E, \epsilon)}{\sqrt{k_{j}( \pm \infty, E)}} c_{j}^{\sigma}( \pm \infty, E, \epsilon)\left(1-e^{i\left(r_{j}^{\sigma}( \pm, x, E)\right) / \epsilon^{2}}\right) \int_{0}^{x} \frac{\partial}{\partial E} k_{j}^{\sigma}(y, E) d y .
\end{aligned}
$$

The third integral contains

$$
\begin{aligned}
g_{3}(x, E, \epsilon) & =\frac{\partial}{\partial E}\left(\frac{Q(E, \epsilon) c_{j}^{\sigma}(x, E, \epsilon)}{\sqrt{k_{j}( \pm \infty, E) k_{j}(x, E)}} \frac{k_{j}( \pm \infty, E)-k_{j}(x, E)}{\sqrt{2 k_{j}( \pm \infty, E)}+\sqrt{2 k_{j}(x, E)}}\right) \\
& -i \frac{Q(E, \epsilon) c_{j}^{\sigma}(x, E, \epsilon)}{\sqrt{k_{j}( \pm \infty, E) k_{j}(x, E)}} \frac{k_{j}( \pm \infty, E)-k_{j}(x, E)}{\sqrt{2 k_{j}( \pm \infty, E)}+\sqrt{2 k_{j}(x, E)}} \int_{0}^{x} \frac{\partial}{\partial E} k_{j}^{\sigma}(y, E) d y .
\end{aligned}
$$

By Lemma 3.1 and the condition $\nu>1 / 2$, each of these functions $g_{j}(x, E, \epsilon)$ satisfies the following bound, uniformly in $E$,

$$
g_{j}(x, E, \epsilon)=O\left(<x>^{-\nu}\right) \in L^{2}(\mathbb{R})
$$

Therefore, we can estimate the $L^{2}$ norm of the corresponding expression (7.11) by

$$
\int_{\mathbb{R}}\left|\int_{\Delta} g_{j}(x, E, \epsilon) e^{-i\left(\int_{0}^{x} k_{j}^{\sigma}(y, E) d y+t E\right) / \epsilon^{2}} d E\right|^{2} d x \leq C_{1}(\epsilon)
$$

where $C_{1}(\epsilon)$ is a finite constant that is independent of $t$. This finishes the proof.

Proof of Proposition 3.1: Since the argument is virtually identical to the one presented in [18] and [15, we will be rather sketchy and mainly point out the effects of the parameter $\delta$ and of the non self-adjointness of the generator $F(x, \delta)$. 
Expressing the projector $P(x, \delta)$ as a integral of the resolvent $(F(x, \delta)-z)^{-1}$ along a loop $L$ (or a finite number of such loops) around the set $\sigma_{1}(x, \delta)$ by means of the Riesz formula,

$$
P(x, \delta)=-\frac{1}{2 \pi i} \oint_{L}(F(x, \delta)-z)^{-1} d z,
$$

we get a bound, uniform in $\delta>0$ and $x \in \rho_{\alpha}$,

$$
\|P(x, \delta)\| \leq c
$$

Indeed, for each $x \in \rho_{\alpha}$, we can choose the path $L$ uniformly in $\delta$ by hypothesis. The existence of the limits $F( \pm \infty, \delta)$ allows us actually to consider only a finite number of distinct loops a finite distance $g / 4$ away from spectrum of $F(x, \delta)$, for all $(x, \delta)$. Also, uniformly in $\delta>0$,

$$
\left\|(F(x, \delta)-z)^{-1}\right\| \leq c
$$

for $z$ on the corresponding loop $L$, since $|\operatorname{det}(F(x, \delta)-z)| \geq(g / 4)^{n}$ and $F(x, \delta)$ is uniformly bounded. By a similar argument, using hypothesis $\mathbf{H 4}$, we get, uniformly in $\delta$

$$
\|P(x, \delta)-P( \pm \infty, \delta)\| \leq \frac{c}{<x>^{2+\nu}}
$$

as $x \rightarrow \pm \infty$ in $\rho_{\alpha}$. With the notation ' for $\frac{\partial}{\partial x}$, we get from (17.15),

$$
P^{\prime}(x, \delta)=\frac{1}{2 \pi i} \oint_{L}(F(x, \delta)-z)^{-1} F^{\prime}(x, \delta)(F(x, \delta)-z)^{-1} d z .
$$

Thus, hypothesis $\mathbf{H} 4$ yields, uniformly in $\delta$,

$$
\left\|P^{\prime}(x, \delta)\right\| \leq \frac{c}{<x>^{2+\nu}}
$$

and a similar uniform estimate for $K(x, \delta)=\left[P^{\prime}(x, \delta), P(x, \delta)\right]$,

$$
\|K(x, \delta)\| \leq \frac{c}{<x>^{2+\nu}}
$$

The operator $K$ is the generator of the intertwining operator $W$ defined by

$$
W^{\prime}\left(x, x_{0}, \delta\right)=K(x, \delta) W\left(x, x_{0}, \delta\right), \quad \text { with } \quad W\left(x_{0}, x_{0}, \delta\right)=\mathbb{I} .
$$

It satisfies

$$
W\left(x, x_{0}, \delta\right) P\left(x_{0}, \delta\right)=P(x, \delta) W\left(x, x_{0}, \delta\right),
$$

for all $(x, \delta)$ (including $x= \pm \infty$ ).

Following [19], we construct a hierarchy of generators. Let $F_{0}(x, \delta)=F(x, \delta)$, $P_{0}(x, \delta)=P(x, \delta)$, and $K_{0}(x, \delta)=K(x, \delta)$. For $q \in \mathbf{N}^{*}$, we inductively define

$$
F_{q}(x, \delta, \epsilon)=F(x, \delta)-\epsilon^{2} K_{q-1}(x, \delta, \epsilon),
$$


assuming $\epsilon$ is small enough so that the spectrum of $F_{q}$ is separated into two disjoint parts corresponding to those of $F$. We define $P_{q}(x, \delta, \epsilon)$ to be the spectral projector for $F_{q}$ corresponding to $P(x, \delta)$ as $\epsilon \rightarrow 0$ by perturbation theory. Then,

$$
K_{q}(x, \delta, \epsilon)=\left[P_{q}^{\prime}(x, \delta, \epsilon), P_{q}(x, \delta, \epsilon)\right] .
$$

Sections II.A and II.B of [19] and (7.16) and (7.17) show that there exist constants $\epsilon^{*}>0$, $r>0, \Gamma>0$ and $c>0$, all independent of $\delta>0$, such that for all $\epsilon<\epsilon^{*}$, all $x \in \mathbb{R}$, and $q=q^{*}=\left[r / \epsilon^{2}\right]$,

$$
\begin{aligned}
& \left\|K_{q^{*}-1}(x, \delta, \epsilon)\right\| \leq \frac{c}{<x>^{2+\nu}} \\
& \left\|K_{q^{*}}(x, \delta, \epsilon)-K_{q^{*}-1}(x, \delta, \epsilon)\right\| \leq c \frac{e^{-\Gamma / \epsilon^{2}}}{<x>^{2+\nu}} .
\end{aligned}
$$

We define

$$
\begin{aligned}
& F_{*}(x, \delta, \epsilon)=F_{q^{*}}(x, \delta, \epsilon)=F(x, \delta)-\epsilon K_{q^{*}-1}(x, \delta, \epsilon), \\
& P_{*}(x, \delta, \epsilon)=P_{q^{*}}(x, \delta, \epsilon) \\
& K_{*}(x, \delta, \epsilon)=K_{q^{*}}(x, \delta, \epsilon)
\end{aligned}
$$

and the evolution operators $W_{*}$ and $\Xi_{*}$ by

$$
W_{*}^{\prime}\left(x, x_{0}, \delta, \epsilon\right)=K_{*}(x, \delta, \epsilon) W_{*}\left(x, x_{0}, \delta, \epsilon\right), \quad \text { with } \quad W\left(x_{0}, x_{0}, \delta, \epsilon\right)=I I,
$$

and

$$
\begin{array}{r}
i \epsilon^{2} \Xi_{*}^{\prime}\left(x, x_{0}, \delta, \epsilon\right)=W_{*}\left(x_{0}, x, \delta, \epsilon\right) F_{*}(x, \delta, \epsilon) W_{*}\left(x, x_{0}, \delta, \epsilon\right) \Xi_{*}\left(x, x_{0}, \delta, \epsilon\right), \\
\text { with } \quad \Xi_{*}\left(x_{0}, x_{0}, \delta, \epsilon\right)=I I .
\end{array}
$$

The intertwining property (7.18) still holds with the $*$ indices. Therefore, $\Xi_{*}$ satisfies

$$
\left[\Xi_{*}\left(x, x_{0}, \delta, \epsilon\right), P_{*}\left(x_{0}, \delta, \epsilon\right)\right] \equiv 0, \quad \text { for all } \quad x \in \mathbb{R} .
$$

It follows from the definitions that the operator

$$
V_{*}\left(x, x_{0}, \delta, \epsilon\right)=W_{*}\left(x, x_{0}, \delta, \epsilon\right) \Xi_{*}\left(x, x_{0}, \delta, \epsilon\right)
$$

satisfies

$$
i \epsilon^{2} V_{*}^{\prime}\left(x, x_{0}, \delta, \epsilon\right)=\left(F_{*}(x, \delta, \epsilon)+i \epsilon^{2} K_{*}(x, \delta, \epsilon)\right) V_{*}\left(x, x_{0}, \delta, \epsilon\right)
$$

and

$$
V_{*}\left(x, x_{0}, \delta, \epsilon\right) P_{*}\left(x_{0}, \delta, \epsilon\right)=P_{*}(x, \delta, \epsilon) V_{*}\left(x, x_{0}, \delta, \epsilon\right) .
$$

Moreover,

$$
\begin{aligned}
U_{\epsilon}\left(x, x_{0}, \delta\right) & -V_{*}\left(x, x_{0}, \delta, \epsilon\right)= \\
& i \int_{x_{0}}^{x} V_{*}\left(x_{0}, y, \delta, \epsilon\right)\left(K_{q^{*}}(y, \delta, \epsilon)-K_{q^{*}-1}(y, \delta, \epsilon)\right) U_{\epsilon}\left(y, x_{0}, \delta\right) d y .
\end{aligned}
$$


The proposition will follow from

$$
U_{\epsilon}\left(x, x_{0}, \delta\right)-V_{*}\left(x, x_{0}, \delta, \epsilon\right)=O\left(e^{-\Gamma / \epsilon^{2}}\right),
$$

(7.22) and

$$
\lim _{x \rightarrow \pm \infty} P_{*}(x, \delta, \epsilon)=P( \pm \infty, \delta)
$$

due to (7.19) and (7.20).

To prove (7.24), we first prove that $V_{*}$ is uniformly bounded in $x, x_{0}$, and $\epsilon$. The analysis leading to Lemma 3.1 implies that $U_{\epsilon}$ is uniformly bounded in $x, x_{0}$, and $\epsilon$. This property is a consequence on the fact that the eigenvalues of $F$ are simple and real, so that the decomposition (3.11) holds and the singular exponential factors are phases. Note that the lack of orthogonality of the eigenprojectors of $F(x, \delta)$ makes the bound on $U_{\epsilon}$ dependent on $\delta$.

Choose $B(\delta)>0$, such that $\left\|U_{\epsilon}\left(x, x_{0}, \delta\right)\right\| \leq B(\delta)$. From (7.23) we get the inequality

$$
\left\|V_{*}\left(x, x_{0}, \delta, \epsilon\right)\right\| \leq B(\delta)\left(1+\int_{x_{0}}^{x} \sup _{y_{0}, y}\left\|V_{*}\left(y, y_{0}, \delta, \epsilon\right)\right\| \frac{C e^{-\Gamma / \epsilon^{2}}}{\langle x\rangle^{2+\nu}}\right) d x
$$

for some $C$. This implies that for some $\widetilde{C}$, the quantity $v(\epsilon, \delta)=\sup _{x_{0}, x}\left\|V_{*}\left(x, x_{0}, \delta, \epsilon\right)\right\|$ satisfies

$$
v(\epsilon, \delta) \leq B(\delta)\left(1+\widetilde{C} v(\epsilon, \delta) e^{-\Gamma / \epsilon^{2}}\right)
$$

This implies

$$
v(\epsilon, \delta) \leq \frac{B(\delta)}{1-\widetilde{C} B(\delta) e^{-\Gamma / \epsilon^{2}}} \leq \widetilde{v}(\delta),
$$

where $\widetilde{v}(\delta)$ is uniformly bounded for sufficiently small $\epsilon$.

We now use (7.23) again to see that

$$
\begin{aligned}
\left\|U_{\epsilon}\left(x, x_{0}, \delta\right)-V_{*}\left(x, x_{0}, \delta, \epsilon\right)\right\| & \leq \widetilde{v}(\delta) \int_{\mathbb{R}} \frac{B(\delta) C e^{-\Gamma / \epsilon^{2}}}{\langle x\rangle^{2+\nu}} d x \\
& \leq \widetilde{C}_{1} e^{-\Gamma / \epsilon^{2}}
\end{aligned}
$$

This proves (17.24) and completes the proof of the proposition.

Proof of Lemma 3.3; Degenerate perturbation theory for self-adjoint matrices and hypothesis $\mathbf{H} \mathbf{5}$ (see [14]) show that there exist $f(z, \delta)$ and $\rho(z, \delta)$, analytic in $z$ for fixed $\delta$, and $C^{1}$ as functions of $(z, \delta)$, such that

$$
\begin{aligned}
& e_{j}(z, \delta)=f(z, \delta)-\frac{1}{2} \sqrt{\rho(z, \delta)} \\
& e_{n}(z, \delta)=f(z, \delta)+\frac{1}{2} \sqrt{\rho(z, \delta)}
\end{aligned}
$$

where, as $(z, \delta) \rightarrow(0,0)$,

$$
f(z, \delta)=f(0,0)+O(|z|+\delta)=e_{c}+O(|z|+\delta), \quad \text { and } \quad \rho(z, \delta)=O\left(|z|^{2}+\delta^{2}\right) .
$$


Moreover, $\rho(z, \delta)$ has two simple zeros, the complex crossing points, $z_{0}(\delta)$ and $\bar{z}_{0}(\delta)$ that have $z_{0}(\delta)=O(\delta)$. For concreteness, we arbitrarily choose $e_{j}<e_{n}$ on the real axis, although this is irrelevant for the analysis. Thus, by $\mathbf{H 7}$, we can write

$$
\sqrt{2\left(E-e_{j}(z, \delta)\right)}=\sqrt{2(E-f(z, \delta))}\left(1+\frac{\sqrt{\rho(z, \delta)}}{2(E-f(z, \delta))}\right)^{1 / 2},
$$

where $(E-f(z, \delta))$ and its inverse are analytic in $\rho_{\alpha}$, uniformly in $E \in \Delta$. Moreover,

$$
\left(1+\frac{\sqrt{\rho(z, \delta)}}{2(E-f(z, \delta))}\right)^{1 / 2}=1+\frac{1}{2} \frac{\sqrt{\rho(z, \delta)}}{2\left(E-e_{c}\right)}+O\left(|z|^{2}+\delta^{2}\right) .
$$

Therefore, since $\sqrt{2(E-f(z, \delta))}$ is analytic, and we can choose the loop $\zeta$ encircling $z_{0}(\delta)$ or $\bar{z}_{0}(\delta)$ to satisfy $|\zeta|=O(\delta)$, we see that

$$
\int_{\zeta} \sqrt{2\left(E-e_{j}(z, \delta)\right)} d z=\frac{1}{\sqrt{2\left(E-e_{c}\right)}} \int_{\zeta} \frac{\sqrt{\rho(z, \delta)}}{2} d z+O\left(\delta^{3}\right)
$$

and

$$
\int_{\zeta} \frac{\sqrt{\rho(z, \delta)}}{2} d z=O\left(\delta^{2}\right)
$$

In these two expressions, $\int_{\zeta} \frac{\sqrt{\rho(z, \delta)}}{2} d z=\int_{\zeta} e_{j}(z, \delta) d z$ due to the analyticity of $f$ in (7.25). Taking the imaginary part yields the first statement of the lemma. Note that we do not have to worry about sign issues because Theorem 3.1 ensures the decay rate, $\operatorname{Im} \int_{\zeta} \sqrt{2\left(E-e_{j}(z, \delta)\right)} d z$, is positive.

The two other statements follow from similar considerations for the integrals

$$
\begin{aligned}
\frac{\partial}{\partial E} \operatorname{Im} \int_{\zeta} \sqrt{2\left(E-e_{j}(z, \delta)\right)} d z & =\operatorname{Im} \int_{\zeta} \frac{1}{\sqrt{2\left(E-e_{j}(z, \delta)\right)}} d z \quad \text { and } \\
\frac{\partial^{2}}{\partial E^{2}} \operatorname{Im} \int_{\zeta} \sqrt{2\left(E-e_{j}(z, \delta)\right)} d z & =-\operatorname{Im} \int_{\zeta} \frac{1}{\left(2\left(E-e_{j}(z, \delta)\right)\right)^{3 / 2}} d z .
\end{aligned}
$$

\section{Proof of Lemma 5.1;}

Consider first the minimization of the negative of the real part of the exponent.

Since $\gamma_{j}(E)$ tends to zero with $\delta$ (absent in the notation), if $\delta$ is small enough, we must look for minima in a neighborhood of $E_{0}$ that satisfy the equation

$$
\alpha^{\prime}(E)=g\left(E-E_{0}\right)+\operatorname{Im} \gamma_{j}^{\prime}(E)+O\left(E-E_{0}\right)^{2}=0
$$


We consider the absolute minimum $E^{*}$ of $\alpha$ and assume it is unique. By Lemma 3.3. $\operatorname{Im} \gamma_{j}^{\prime}(E)<0$, so $E^{*}>E_{0}$. Note that $E^{*}$ does not depend on $x$ or $t$. Also, $\operatorname{Im} \gamma_{j}^{(n)}(E)=o(\delta)$, $n=0,1,2$, uniformly in $E$. So, we can assume $E^{*}$ is non-degenerate since

$$
\alpha^{\prime \prime}\left(E^{*}\right)=g+\operatorname{Im} \gamma_{j}^{\prime \prime}\left(E^{*}\right)+O\left(E^{*}-E_{0}\right)>0 .
$$

In terms of the variable $k \in\left[k_{1}, k_{2}\right]$, we view $T$ as the (scaled) inverse Fourier transform of the function

$$
R(k, t, \epsilon)=\sqrt{2 \pi \epsilon^{2}} e^{-\alpha(E(k)) / \epsilon^{2}} \tilde{P}(E(k), \epsilon) \sqrt{k} e^{-i \kappa(E(k)) / \epsilon^{2}} e^{-i t\left(k^{2} / 2+e(\infty)\right) / \epsilon^{2}} \chi_{\left[k_{1}, k_{2}\right]}(k),
$$

where $\chi_{S}(\cdot)$ is the characteristic function of the set $S$. That is

$$
T(x, t, \epsilon)=\left(\mathcal{F}_{\epsilon}^{-1} R(\cdot, t, \epsilon)\right)(x),
$$

where $\mathcal{F}_{\epsilon}$ is defined by

$$
\left(\mathcal{F}_{\epsilon} g\right)(x)=\frac{1}{\sqrt{2 \pi \epsilon^{2}}} \int_{\mathbb{R}} g(k) e^{-i k x / \epsilon^{2}} d k
$$

With the variable $k \in\left[k_{1}, k_{2}\right]$ we have

$$
\left.\frac{\partial^{2}}{\partial k^{2}} \alpha(E(k))\right|_{k^{*}}=k^{* 2} \alpha^{\prime \prime}\left(E^{*}\right),
$$

and expanding around $k^{*}$,

$$
\begin{aligned}
T(\epsilon, x, t)= & e^{-\alpha\left(E^{*}\right) / \epsilon^{2}} \\
& \times \int_{\left[k_{1}, k_{2}\right]} \sqrt{k} \tilde{P}(E(k), \epsilon) e^{-\frac{\frac{\partial^{2}}{\left.\partial k^{2} \alpha(E(k))\right|_{k^{*}}}}{2 \epsilon^{2}}\left(k-k^{*}\right)^{2}} e^{O\left(\left(k-k^{*}\right)^{3}\right) / \epsilon^{2}} e^{-i \beta(k, x, t) / \epsilon^{2}} d k,
\end{aligned}
$$

where the negative of the imaginary part of the exponent is denoted by

$$
\beta(k, x, t)=t\left(\frac{k^{2}}{2}+e_{2}(\infty)\right)+\kappa(E(k))-x k .
$$

We now introduce $\mu(\epsilon)=\epsilon^{s}>0$, with $2 / 3<s<1$. It goes to zero in such a way that

$$
\mu(\epsilon) / \epsilon>>1 \text { and } \mu(\epsilon)^{3} / \epsilon^{2}<<1 .
$$

Because $E^{*}$ is a unique absolute minimum, the behavior of $\alpha(E)$ close to $E^{*}$, and the assumption (5.2) on $P$, we can reduce the integration range in $T$ to $\left[k^{*}-\mu(\epsilon), k^{*}+\mu(\epsilon)\right]$ at the expense of a relative error whose $L^{2}$ norm is of order $O\left(\epsilon^{\infty}\right)$, uniformly $t$. More precisely,

$$
T(x, t, \epsilon)=\left(\left(\mathcal{F}_{\epsilon}^{-1}\left(R_{1}+R_{2}\right)\right)(\cdot, t, \epsilon)\right)(x),
$$

where

$$
\begin{aligned}
& R_{1}(k, t, \epsilon)=\chi_{\left[k^{*}-\mu(\epsilon), k^{*}+\mu(\epsilon)\right]}(k) R(k, t, \epsilon), \quad \text { and } \\
& R_{2}(k, t, \epsilon)=\chi_{\left[k^{*}-\mu(\epsilon), k^{*}+\mu(\epsilon)\right]^{C}}(k) R(k, t, \epsilon) .
\end{aligned}
$$


For some $a^{*}>0$ and $r>0$,

$$
\left|R_{2}(k, t, \epsilon)\right| \leq r e^{-\alpha\left(E^{*}\right) / \epsilon^{2}} e^{-a^{*}(\mu(\epsilon) / \epsilon)^{2}} \epsilon|\sqrt{k} \tilde{P}(E(k), \epsilon)| .
$$

Hence, by the Parseval identity, uniformly in $t$, we have

$$
\left.\| \mathcal{F}_{\epsilon}^{-1}\left(R_{2}\right)(\cdot, t, \epsilon)\right) \|=\left\{\int_{\left[k^{*}-\mu(\epsilon), k^{*}+\mu(\epsilon)\right]^{C}}\left|R_{2}(k, t, \epsilon)\right|^{2} d k\right\}^{1 / 2}=O\left(e^{-\alpha\left(E^{*}\right) / \epsilon^{2}} \epsilon^{\infty}\right) .
$$

In the remaining integral containing $R_{1}$, we further estimate

$$
e^{O\left(k-k^{*}\right)^{3} / \epsilon^{2}}=1+O\left(\mu(\epsilon)^{3} / \epsilon^{2}\right)=1+O\left(\epsilon^{3 s-2}\right),
$$

and

$$
\sqrt{k} \tilde{P}(E(k), \epsilon)=\sqrt{k^{*}} \tilde{P}\left(E^{*}, \epsilon\right)+O(\mu(\epsilon))=\sqrt{k^{*}} P\left(E^{*}, \epsilon\right)+O\left(\epsilon^{s}+\epsilon^{2}\right) .
$$

The contribution of order $\epsilon^{2}$ comes from the error in the computation of the coefficient $c_{n}^{-}$. Using the Parseval identity again with uniform bounds on the exponential factors of $R_{1}$, we see that the contribution to $T$ coming from the error term $O\left(\epsilon^{s}\right)$ is bounded uniformly in $t$ in the $L^{2}\left(\mathbb{R}_{x}\right)$ norm by $O\left(e^{-\alpha\left(E^{*}\right) / \epsilon^{2}} \epsilon^{1+2 s}\right)$. Similarly, the error term stemming from (77.26) yields an error in the $L^{2}\left(\mathbb{R}_{x}\right)$ norm of order $O\left(e^{-\alpha\left(E^{*}\right) / \epsilon^{2}} \epsilon^{4 s-1}\right)$.

To compute the leading term, we expand $\beta(\cdot, x, t)$ around $k^{*}$ as

$$
\begin{aligned}
\beta(k, x, t) & =t E^{*}+\kappa\left(E^{*}\right)-x k^{*} \\
& +\left(k-k^{*}\right)\left(k^{*} t+\left.\frac{\partial}{\partial k} \kappa(E(k))\right|_{k^{*}}-x\right) \\
& +\frac{\left(k-k^{*}\right)^{2}}{2}\left(t+\left.\frac{\partial^{2}}{\partial k^{2}} \kappa(E(k))\right|_{k^{*}}\right) \\
& +\left.\frac{\left(k-k^{*}\right)^{3}}{6} \frac{\partial^{3}}{\partial k^{3}} \kappa(E(k))\right|_{\tilde{k}},
\end{aligned}
$$

where $\tilde{k}$ lies between $k$ and $k^{*}$, and the third derivative is independent of $t$ and $x$. The last term in (17.27) gives rise to a contribution which is of order $O\left(e^{-\alpha\left(E^{*}\right) / \epsilon^{2}} \epsilon^{4 s-1}\right)$ in the $L^{2}\left(\mathbb{R}_{x}\right)$ norm, uniformly in $t$, as above.

Therefore, in the $L^{2}$ sense,

$$
\begin{aligned}
T(\epsilon, x, t)= & e^{-\alpha\left(E^{*}\right) / \epsilon^{2}} e^{-i\left(t E^{*}+\kappa\left(E^{*}\right)-x k^{*}\right) / \epsilon^{2}} \\
& \times\left\{\int_{\left[k^{*}-\mu(\epsilon), k^{*}+\mu(\epsilon)\right]} \sqrt{k^{*}} P\left(E^{*}, \epsilon\right) e^{\left.-i \frac{\left(k-k^{*}\right)}{\epsilon^{2}}\left(k^{*} t+\left.\frac{\partial}{\partial k} \kappa(E(k))\right|_{k^{*}}\right)-x\right)}\right. \\
& \left.\times e^{-\frac{\left(k-k^{*}\right)^{2}}{2 \epsilon^{2}}\left(\left.\frac{\partial^{2}}{\partial k^{2}} \alpha(E(k))\right|_{k^{*}}+i\left(t+\left.\frac{\partial^{2}}{\partial k^{2}} \kappa(E(k))\right|_{k^{*}}\right)\right)} d k+O\left(\epsilon^{p}\right)+O\left(\epsilon^{\infty}\right)\right\},
\end{aligned}
$$

where $p=\min (1+2 s, 4 s-1) \in(0,3)$ can be chosen arbitrarily close to 3 . Again, at the cost of an error whose $L^{2}$ norm is $O\left(e^{-\alpha\left(E^{*}\right) / \epsilon^{2}} \epsilon^{\infty}\right)$, uniformly in $t$, we can extend the interval 
of integration to the whole real line and compute the Gaussian integral explicitly according to the formula (for $\operatorname{Re} M>0$ )

$$
\int_{-\infty}^{\infty} \sqrt{k^{*}} e^{-\left(M\left(k-k^{*}\right)^{2} / 2+i N\left(k-k^{*}\right)\right) / \epsilon^{2}} d k=\frac{\epsilon}{\sqrt{k^{*}}} e^{-\frac{N^{2}}{2 \epsilon^{2} M}}\left(\sqrt{\frac{2 \pi}{M}}\left(k^{*}-i N / M\right)\right) .
$$

We then get the result with

$$
\begin{aligned}
M & =\left.\frac{\partial^{2}}{\partial k^{2}} \alpha(E(k))\right|_{k^{*}}+i\left(t+\left.\frac{\partial^{2}}{\partial k^{2}} \kappa(E(k))\right|_{k^{*}}\right), \quad \text { and } \\
N & =k^{*} t+\left.\frac{\partial}{\partial k} \kappa(E(k))\right|_{k^{*}}-x
\end{aligned}
$$

Proof of Lemma [5.2; The first assertion is straightforward. The second follows from the identity

$$
\begin{aligned}
\varphi_{0}\left(A_{+}(t), B_{+}(t), \epsilon^{2}, a_{+}(t), \eta_{+}(t), x\right) x= & \varphi_{0}\left(A_{+}(t), B_{+}(t), \epsilon^{2}, a_{+}(t), \eta_{+}(t), x\right)\left(x-a_{+}(t)\right) \\
& +\varphi_{0}\left(A_{+}(t), B_{+}(t), a_{+}(t), \epsilon^{2}, \eta_{+}(t), x,\right) a_{+}(t) .
\end{aligned}
$$

The first term is $O(\epsilon)$ in $L^{2}(\mathbb{R})$ by scaling, and the second is of order $a_{+}(t)=k^{*} t(1+O(1 /|t|))$ for $|t|$ large. We insert this in the first part of the lemma to obtain the second part as $t \rightarrow \pm \infty$.

\section{References}

[1] Benchaou M., and Martinez A.: Estimations Exponentielles en Théorie de la Diffusion des Opérateurs de Schrödinger Matriciels. Ann. Inst. H. Poincaré Sect. A 71, 561-594 (1999).

[2] Betz V., and Teufel S.: Precise coupling terms in adiabatic quantum evolution. Preprint, mp_arc 04-102.

[3] Betz V., and Teufel S.: Private Communication.

[4] Coker, D. F., and Xiao, L: Methods for Molecular-Dynamics with Nonadiabtic Transitions. J. Chem. Phys. 102, 496-510 (1995).

[5] Fedoriuk, M.: Méthodes Asymptotiques pour les Equations Différentielles Ordinaires Linéaires, Mir, Moscou, 1987.

[6] Fedoriuk, M.: Analysis I, in Encyclopaedia of Mathematical Sciences, Vol 13, R.V.Gamkrelidze, ed. Springer-Verlag Berlin Heidelberg New York, 1989

[7] Hagedorn, G. A.: Proof of the Landau-Zener Formula in an Adiabatic Limit with Small Eigenvalue Gaps, Commun. Math. Phys. 136, $433-449$ (1991). 
[8] Hagedorn, G. A.: Molecular Propagation Through Electronic Eigenvalue Crossings, Memoirs Amer. Math. Soc. 111 (536), (1994).

[9] Hagedorn, G. A.: Raising and lowering operators for semiclassical wave packets. Ann. Phys. 269, 77-104 (1998).

[10] Hagedorn G. A. and Joye A.: Landau-Zener Transitions Through Small Electronic Eigenvalue Gaps in the Born-Oppenheimer Approximation. Ann. Inst. H. Poincaré, Phys. Théor. 68, 85-134 (1998)

[11] Hagedorn G. A. and Joye A.: Molecular Propagation Through Small Avoided Crossings of Electron Energy Levels. Rev. Math. Phys. 11, 41-101 (1999)

[12] Hagedorn, G. A., and Joye, A.: A Time-Dependent Born-Oppenheimer Approximation with Exponentially Small Error Estimates, Commun. Math. Phys. 223, 583-626 (2001).

[13] Hagedorn, G. A., and Joye, A.: "Time Development of Exponentially Small Non-Adiabatic Transitions" Commun. Math. Phys., to appear.

[14] Joye, A.: Proof of the Landau-Zener Formula. Asymptotic Analysis 9, 209-258 (1994).

[15] Joye, A.: Exponential asymptotics in a singular limit for $n$-level scattering systems. SIAM J. Math. Anal. 28, 669-703 (1997).

[16] Joye, A., Pfister, C.-E. : Complex WKB Method for 3-Level Scattering Systems. Asymptotic Anal. 23, 91-109 (2000).

[17] Joye, A., Kunz, H., Pfister, C.-E.: Exponential Decay and Geometric Aspect of Transition Probabilities in the Adiabatic Limit. Ann. Phys. 208, 299-332 (1991).

[18] Joye, A., Pfister, C.-E.: Semi-Classical Asymptotics beyond All Orders for Simple Scattering Systems. SIAM J. Math. Anal. 26, 944-977 (1995).

[19] Joye, A., Pfister, C.-E.: Superadiabatic Evolution and Adiabatic Transition Probability between Two Non-degenerate Levels Isolated in the Spectrum. J. Math. Phys. 34, 454-479 (1993).

[20] T. Kato: Perturbation Theory for Linear Operators. Springer-Verlag Berlin Heidelberg New York 1980.

[21] Martin Ph.-A. and Nenciu G.: Semiclassical Inelastic S-Matrix for OneDimensional N-States Systems. Rev. Math. Phys. 7, 193-242 (1995)

[22] Martinez, A. and Sordoni, V.: On the Time-Dependent Born-Oppenheimer Approximation with Smooth Potential. Preprint mp_arc 01-37.

[23] Martinez, A. and Sordoni, V.: A general reduction scheme for the timedependent Born-Oppenheimer approximation. C.R.A.S. 334, 185-188 (2002). 
[24] Nenciu, G., and Sordoni, V.: Semiclassical limit for multistate Klein-Gordon systems: almost invariant subspaces and scattering theory.

Preprint mp_arc 01-36.

[25] Ramond, T.: Semiclassical Study of Quantum Scattering on the Line. Commun. Math. Phys. 177, 221-254 (1996).

[26] Rousse, V.: Landau-Zener Transitions for Eigenvalue Avoided Crossings in the Adiabatic and Born-Oppenheimer Approximations. Asymptotic Analysis 37, 293328 (2004).

[27] Tully, J. C.: Molecular Dynamics with Electronic Transitions. J. Chem. Phys. 93, 1061-1071 (1990).

[28] Webster, F., Rossky, P. J., and Friesner, R. A.: Nonadiabatic Processes in Condensed Matter: Semi-Classical Theory and Implementation. Comp. Phys. Commun. 63, 494-522 (1991). 